УДК 517.555

\author{
A. I. BANDURA
}

\title{
COMPOSITION, PRODUCT AND SUM OF ANALYTIC FUNCTIONS OF BOUNDED $L$-INDEX IN DIRECTION IN THE UNIT BALL
}

\begin{abstract}
A. I. Bandura. Composition, product and sum of analytic functions of bounded L-index in direction in the unit ball, Mat. Stud. 50 (2018), 115-134.

In this paper, we investigate a composition of entire function of one variable and analytic function in the unit ball. There are obtained conditions which provide equivalence of boundedness of $L$-index in a direction for such a composition and boundedness of $l$-index of initial function of one variable, where the continuous function $L: \mathbb{B}^{n} \rightarrow \mathbb{R}_{+}$is constructed by the continuous function $l: \mathbb{C} \rightarrow \mathbb{R}_{+}$. We present sufficient conditions for boundedness of $L$-index in the direction for sum and for product of functions analytic in the unit ball.

The class of analytic functions in the unit ball having bounded $L$-index in direction is very wide because it contains all analytic functions with bounded multiplicities of zeros on every complex line $\left\{z^{0}+t \mathbf{b}: t \in \mathbb{C}\right\}$. It is a statement of proved existence theorem. In the one-dimensional case these results are new for functions analytic in the unit disc.
\end{abstract}

1. Introduction. Let $\mathbb{B}^{n}=\left\{z \in \mathbb{C}^{n}:|z|<1\right\}$. The paper is a continuation of $[2,6,7]$. There was generalized a concept of $L$-index boundedness in a direction for a class of analytic functions in the unit ball (see the definition below), including many criteria of $L$-index boundedness in the direction, where $L: \mathbb{B}^{n} \rightarrow \mathbb{R}_{+}$is a continuous function.

In this paper, we will apply some obtained results from [6] to deduce sufficient conditions of $L$-index boundedness in direction for some composite analytic functions in the unit ball and sum of these functions. Also we prove that analytic functions in the unit ball has bounded $L$ index in any direction for compactly embedded domain in the unit ball. Among other results we show that for any analytic function $F: \mathbb{B}^{n} \rightarrow \mathbb{C}$ with bounded multiplicities of zeros on every complex line $\left\{z^{0}+t \mathbf{b}: t \in \mathbb{C}\right\}$ and any direction $\mathbf{b} \in \mathbb{C}^{n} \backslash\{0\}$ there exists a positive continuous function $L: \mathbb{B}^{n} \rightarrow \mathbb{R}_{+}$such that $F$ is of bounded $L$-index in the direction $\mathbf{b}$. Mostly obtained results are also new for functions analytic in the unit disc.

Note that investigation of properties of analytic functions having bounded $L$-index in direction is very important in view of analytic theory of differential equations. These functions have regular behavior, uniform distribution of zeros in some sense and its growth estimates $[15,24]$. It is known many various conditions providing index boundedness for every analytic solutions of some ordinary and partial differential equations and its system [14,15,24,27].

2010 Mathematics Subject Classification: 30A05, 30H99, 32A10, 32A17, 32A37.

Keywords: bounded index; bounded $L$-index in direction; analytic function; unit ball; composite function; bounded $l$-index; sum; existence theorem.

doi:10.15330/ms.50.2.115-134

(C) A. I. Bandura, 2018 
Let $\mathbb{D}=\{t \in \mathbb{C}:|t|<1\}, L: \mathbb{B}^{n} \rightarrow \mathbb{R}_{+}$be a continuous function, $\mathbf{b}=\left(b_{1}, \ldots, b_{n}\right) \in$ $\mathbb{C}^{n} \backslash\{\mathbf{0}\}$ be a fixed direction, where $\mathbf{0}=(0, \ldots, 0), \mathbf{1}=(1, \ldots, 1)$. For $z \in \mathbb{B}^{n}$ we denote $D_{z}=\left\{t \in \mathbb{C}:|t| \leq \frac{1-|z|}{|\mathbf{b}|}\right\}$,

$$
\lambda_{\mathbf{b}}(\eta)=\sup _{z \in \mathbb{B}^{n}} \sup _{t_{1}, t_{2} \in D_{z}}\left\{\frac{L\left(z+t_{1} \mathbf{b}\right)}{L\left(z+t_{2} \mathbf{b}\right)}:\left|t_{1}-t_{2}\right| \leq \frac{\eta}{\min \left\{L\left(z+t_{1} \mathbf{b}\right), L\left(z+t_{2} \mathbf{b}\right)\right\}}\right\} .
$$

The notation $Q_{\mathbf{b}}\left(\mathbb{B}^{n}\right)$ stands for the class of positive continuous functions $L: \mathbb{B}^{n} \rightarrow \mathbb{R}_{+}$ satisfying

$$
(\forall \eta \in[0, \beta]): \quad \lambda_{\mathbf{b}}(\eta)<+\infty
$$

and

$$
L(z)>\frac{\beta|\mathbf{b}|}{1-|z|},
$$

where $\beta>1$ is some constant. If $n=1$ then $Q(\mathbb{D}) \equiv Q_{1}\left(\mathbb{B}^{1}\right)$ and $\lambda(\eta) \equiv \lambda_{1}(\eta)$.

Similarly, $Q_{\mathrm{b}}^{n}$ stands for the class of positive continuous functions $L: \mathbb{C}^{n} \rightarrow \mathbb{R}_{+}$satisfying (1) with

$$
\lambda_{\mathbf{b}}(\eta)=\sup _{z \in \mathbb{C}^{n}} \sup _{t_{1}, t_{2} \in \mathbb{C}}\left\{\frac{L\left(z+t_{1} \mathbf{b}\right)}{L\left(z+t_{2} \mathbf{b}\right)}:\left|t_{1}-t_{2}\right| \leq \frac{\eta}{\min \left\{L\left(z+t_{1} \mathbf{b}\right), L\left(z+t_{2} \mathbf{b}\right)\right\}}\right\} .
$$

2. Composition of entire functions of bounded $L$-index in direction. Analytic function $F: \mathbb{B}^{n} \rightarrow \mathbb{C}$ is called a function of bounded L-index $[5-7,10]$ in a direction $\mathbf{b} \in \mathbb{C}^{n} \backslash\{\mathbf{0}\}$, if there exists $m_{0} \in \mathbb{Z}_{+}$such that for every $m \in \mathbb{Z}_{+}$and for each $z \in \mathbb{B}^{n}$

$$
\frac{\left|\partial_{\mathbf{b}}^{m} F(z)\right|}{m ! L^{m}(z)} \leq \max _{0 \leq k \leq m_{0}} \frac{\left|\partial_{\mathbf{b}}^{k} F(z)\right|}{k ! L^{k}(z)}
$$

where

$$
\partial_{\mathbf{b}}^{0} F(z)=F(z), \partial_{\mathbf{b}} F(z)=\sum_{j=1}^{n} \frac{\partial F(z)}{\partial z_{j}} b_{j}, \quad \partial_{\mathbf{b}}^{k} F(z)=\partial_{\mathbf{b}}\left(\partial_{\mathbf{b}}^{k-1} F(z)\right), \quad k \geq 2 .
$$

There are also papers on analytic functions in the unit ball of bounded L-index in joint variables [4,9]. A connection between these classes is established in [10,11]. The least integer $m_{0}=m_{0}(\mathbf{b})$ satisfying $(3)$ is called the $L$-index in the direction $\mathbf{b}$ of the analytic function $F$ and is denoted by $N_{\mathbf{b}}(F, L)=m_{0}$. If $n=1, \mathbf{b}=1, L=l, F=f$, then $N(f, l) \equiv N_{1}(f, l)$ is called the $l$-index of the function $f$. In the case $n=1$ and $\mathbf{b}=1$ we obtain the definition of an analytic function in the unit disc of bounded $l$-index ([25]). Similarly, entire function $F: \mathbb{C}^{n} \rightarrow \mathbb{C}$ is called a function of bounded L-index in a direction $\mathbf{b} \in \mathbb{C}^{n} \backslash\{\mathbf{0}\}$, if it satisfies (3) for all $z \in \mathbb{C}^{n}$. If $n=1$ and $L=l$ we obtain the definition of bounded $l$-index for entire functions of one variable [20], and if, in addition, $l \equiv 1$ we have the definition of an entire function of bounded index [21]. Theory of entire functions of bounded $L$-index in direction is developed in [15].

There are many papers on various classes of functions of bounded index (see bibliography $[15,24])$. Nevertheless index boundedness of composite entire and analytic functions were considered only in $[16,18,19,22,24]$. In paper [22], there investigated $l$-index boudnedness of composition $f(P(z)$ ), where $f$ is an entire function and $P$ is a polynomial. In [18] there were presented conditions which provide $l$-index boundedness of the function $f(w(z))$, where $f$ is 
a function analytic in the unit disc $\mathbb{D}=\{z \in \mathbb{C}:|z|<1\}, w(z)=\frac{z-z_{0}}{1-z \bar{z}_{0}} e^{i \alpha}, z_{0} \in \mathbb{D}, \alpha \in \mathbb{R}$. The most general result of such type was obtained in [19] for composite analytic function in arbitrary domains in complex plane. M. M. Sheremeta [24, p. 99] also proved that an entire function $f(z)$ has bounded index if and only if the analytic function $f\left(\frac{1}{z}\right)$ in $\mathbb{C} \backslash\{0\}$ has bounded $l$-index with $l(z)=\frac{1}{|z|^{2}}$.

Note that the multidimensional case $[13,16]$ was considered for the composition of two entire functions, where one of them is an entire function of several variables. The most general result is the following

Theorem 1 ([16]). Let $\mathbf{b} \in \mathbb{C}^{n} \backslash\{\mathbf{0}\}, f$ be an entire function in $\mathbb{C}, \Phi$ be an entire function in $\mathbb{C}^{n}$ such that $\partial_{\mathbf{b}} \Phi(z) \neq 0$ and $\left|\partial_{\mathbf{b}}^{j} \Phi(z)\right| \leq K\left|\partial_{\mathbf{b}} \Phi(z)\right|^{j}, K \equiv$ const $>0$, for all $z \in \mathbb{C}^{n}$ and for all $j \leq p$, where $p=N(f, l)$ or $p=N_{\mathbf{b}}(F, L)$, respectively.

Suppose that $l \in Q, l(w) \geq 1, w \in \mathbb{C}$ and $L \in Q_{\mathbf{b}}^{n}, L(z)=\left|\partial_{\mathbf{b}} \Phi(z)\right| l(\Phi(z))$. The entire function $f$ has bounded l-index if and only if the entire function $F(z)=f(\Phi(z))$ has bounded $L$-index in the direction $\mathbf{b}$.

Similar result ([19]) is also known for functions analytic in an arbitrary domain in the complex plane.

Our main theorem is the following

Theorem 2. Let $\mathbf{b} \in \mathbb{C}^{n} \backslash\{\mathbf{0}\}, f: \mathbb{C}^{m} \rightarrow \mathbb{C}$ be an entire function, $\Phi: \mathbb{B}^{n} \rightarrow \mathbb{C}$ be an analytic function, such that $\partial_{\mathbf{b}} \Phi(z) \neq 0$ and

$$
\left|\partial_{\mathbf{b}}^{j} \Phi(z)\right| \leq K\left|\partial_{\mathbf{b}} \Phi(z)\right|^{j}, \quad K \equiv \text { const }>0,
$$

for all $z \in \mathbb{B}^{n}$ and for all $j \leq p$, where $p=N_{\mathbf{1}}(f, l)$ or $p=N_{\mathbf{b}}(F, L)$, respective.

Suppose that $l \in Q_{1}^{m}, l(w) \geq 1\left(w \in \mathbb{C}^{m}\right), L \in Q_{\mathbf{b}}\left(\mathbb{B}^{n}\right), L(z)=\left|\partial_{\mathbf{b}} \Phi(z)\right| l(\underbrace{\Phi(z), \ldots, \Phi(z)}_{m \text { times }})$. The entire function $f$ has bounded l-index in the direction $\mathbf{1}$ if and only if the analytic function $F(z)=f(\underbrace{\Phi(z), \ldots, \Phi(z)}_{m \text { times }})$ has bounded $L$-index in the direction $\mathbf{b}$.

To prove main theorem we need auxiliary propositions. They are analogs of Hayman's Theorem for entire functions and analytic functions in the unit ball. It was firstly proved by W. Hayman ([17]) for entire functions of one variable having bounded index.

Theorem 3 ([12]). Let $\mathbf{b} \in \mathbb{C}^{n} \backslash\{\mathbf{0}\}$ and $L \in Q_{\mathbf{b}}^{n}$. An entire function $F(z)$ has bounded $L$-index in the direction $\mathbf{b}$ if and only if there exist numbers $p \in \mathbb{Z}_{+}, R>0$ and $C>0$ such that for every $z \in \mathbb{C}^{n},|z| \geq R$,

$$
\frac{\left|\partial_{\mathbf{b}}^{p+1} F(z)\right|}{L^{p+1}(z)} \leq C \max \left\{\frac{\left|\partial_{\mathbf{b}}^{k} F(z)\right|}{L^{k}(z)}: 0 \leq k \leq p\right\} .
$$

Theorem $4([5,6])$. Let $\mathbf{b} \in \mathbb{C}^{n} \backslash\{\mathbf{0}\}$ and $L \in Q_{\mathbf{b}}\left(\mathbb{B}^{n}\right)$. An analytic function $F: \mathbb{B}^{n} \rightarrow \mathbb{C}$ is of bounded $L$-index in the direction $\mathbf{b}$ if and only if there exist $p \in \mathbb{Z}_{+}$and $C>0$ such that for every $z \in \mathbb{B}^{n}$ inequality (5) holds.

Proof of Theorem 2. Denote $\nabla f=\partial_{1} f=\sum_{j=1}^{m} \frac{\partial f}{\partial z_{j}}, \nabla^{k} f \equiv \partial_{1}^{k} f$ for $k \geq 2$. Our proof is similar to the proof of the corresponding theorem in [16]. Firstly, we prove that

$$
\partial_{\mathbf{b}}^{k} F(z)=\nabla^{k} f(\Phi(z), \ldots, \Phi(z))\left(\partial_{\mathbf{b}} \Phi(z)\right)^{k}+\sum_{j=1}^{k-1} \nabla^{j} f(\Phi(z), \ldots, \Phi(z)) Q_{j, k}(z),
$$


where

$$
Q_{j, k}(z)=\sum_{\substack{n_{1}+2 n_{2}+\ldots+k n_{k}=k \\ 0 \leq n_{1} \leq j-1}} c_{j, k, n_{1}, \ldots, n_{k}}\left(\partial_{\mathbf{b}} \Phi(z)\right)^{n_{1}}\left(\partial_{\mathbf{b}}^{2} \Phi(z)\right)^{n_{2}} \ldots\left(\partial_{\mathbf{b}}^{k} \Phi(z)\right)^{n_{k}},
$$

and $c_{j, k, n_{1}, \ldots, n_{k}}$ are non-negative integer numbers. We also will show that

$$
\nabla^{k} f(\Phi(z), \ldots, \Phi(z))=\frac{\partial_{\mathbf{b}}^{k} F(z)}{\left(\partial_{\mathbf{b}} \Phi(z)\right)^{k}}+\frac{1}{\left(\partial_{\mathbf{b}} \Phi(z)\right)^{2 k}} \sum_{j=1}^{k-1} \partial_{\mathbf{b}}^{j} F(z)\left(\partial_{\mathbf{b}} \Phi(z)\right)^{j} Q_{j, k}^{*}(z),
$$

where

$$
Q_{j, k}^{*}(z)=\sum_{m_{1}+2 m_{2}+\ldots+k m_{k}=2(k-j)} b_{j, k, m_{1}, \ldots, m_{k}}\left(\partial_{\mathbf{b}} \Phi(z)\right)^{m_{1}}\left(\partial_{\mathbf{b}}^{2} \Phi(z)\right)^{m_{2}} \ldots\left(\partial_{\mathbf{b}}^{k} \Phi(z)\right)^{m_{k}},
$$

and $b_{j, k, m_{1}, \ldots, m_{k}}$ are some integer coefficients.

The validity of formulas (6) and (7) will be checked by the method of mathematical induction. Of course, for $k=1$ equalities (6) and (7) hold. Assume that they are valid for $k=s$. Let us to prove them for $k=s+1$. Evaluate directional derivative in (6)

$$
\begin{gathered}
\partial_{\mathbf{b}}^{s+1} F(z)=\nabla^{s+1} f(\Phi(z), \ldots, \Phi(z))\left(\partial_{\mathbf{b}} \Phi(z)\right)^{s+1}+s \nabla^{s} f(\Phi(z), \ldots, \Phi(z))\left(\partial_{\mathbf{b}} \Phi(z)\right)^{s-1} \partial_{\mathbf{b}}^{2} \Phi(z)+ \\
+\sum_{j=1}^{s-1}\left(\nabla^{j+1} f(\Phi(z), \ldots, \Phi(z)) \partial_{\mathbf{b}} \Phi(z) Q_{j, s}(z)+\nabla^{j} f(\Phi(z), \ldots, \Phi(z)) \partial_{\mathbf{b}} Q_{j, s}(z)\right)= \\
=\nabla^{s+1} f(\Phi(z), \ldots, \Phi(z))\left(\partial_{\mathbf{b}} \Phi(z)\right)^{s+1}+ \\
+\nabla^{s} f(\Phi(z), \ldots, \Phi(z))\left(s\left(\partial_{\mathbf{b}} \Phi(z)\right)^{s-1} \partial_{\mathbf{b}}^{2} \Phi(z)+\partial_{\mathbf{b}} \Phi(z) Q_{s-1, s}(z)\right)+ \\
+\sum_{j=2}^{s-1} \nabla^{j} f(\Phi(z), \ldots, \Phi(z))\left(\partial_{\mathbf{b}} \Phi(z) Q_{j-1, s}(z)+\partial_{\mathbf{b}} Q_{j, s}(z)\right)+\nabla f(\Phi(z), \ldots, \Phi(z)) \partial_{\mathbf{b}} Q_{1, s}(z) .
\end{gathered}
$$

Since

$$
\begin{aligned}
& s\left(\partial_{\mathbf{b}} \Phi(z)\right)^{s-1} \partial_{\mathbf{b}}^{2} \Phi(z)+\sum_{\substack{n_{1}+2 n_{2}+\ldots+s n_{s}=s \\
0 \leq n_{1} \leq s-2}} c_{s-1, s, n_{1}, \ldots, n_{s}}\left(\partial_{\mathbf{b}} \Phi(z)\right)^{n_{1}+1}\left(\partial_{\mathbf{b}}^{2} \Phi(z)\right) \ldots\left(\partial_{\mathbf{b}}^{s} \Phi(z)\right)^{n_{s}}= \\
& =\sum_{\substack{m_{1}+2 m_{2}+\ldots+s m_{s}=s+1 \\
0 \leq m_{1} \leq s-1}} \tilde{c}_{s, s+1, m_{1}, \ldots, m_{s}}\left(\partial_{\mathbf{b}} \Phi(z)\right)^{m_{1}}\left(\partial_{\mathbf{b}}^{2} \Phi(z)\right)^{m_{2}} \ldots\left(\partial_{\mathbf{b}}^{s} \Phi(z)\right)^{m_{s}}=Q_{s, s+1}(z), \\
& \partial_{\mathbf{b}} Q_{1, s}(z)=\sum_{2 n_{2}+\ldots+s n_{s}=s} c_{1, s, 0, n_{2}, \ldots, n_{s}}\left(n_{2}\left(\partial_{\mathbf{b}}^{2} \Phi(z)\right)^{n_{2}-1}\left(\partial_{\mathbf{b}}^{3} \Phi(z)\right)^{n_{3}+1} \ldots\left(\partial_{\mathbf{b}}^{s} \Phi(z)\right)^{n_{s}}+\right. \\
& \left.+\ldots+n_{s}\left(\partial_{\mathbf{b}}^{2} \Phi(z)\right)^{n_{2}}\left(\partial_{\mathbf{b}}^{3} \Phi(z)\right)^{n_{3}} \ldots\left(\partial_{\mathbf{b}}^{s} \Phi(z)\right)^{n_{s}-1} \partial_{\mathbf{b}}^{s+1} \Phi(z)\right)= \\
& =\sum_{2 m_{2}+\ldots+(s+1) m_{s+1}=s+1} \tilde{c}_{1, s+1,0, m_{2}, \ldots, m_{s+1}}\left(\partial_{\mathbf{b}}^{2} \Phi(z)\right)^{m_{2}} \ldots\left(\partial_{\mathbf{b}}^{s} \Phi(z)\right)^{m_{s}} \times \\
& \times\left(\partial_{\mathbf{b}}^{s+1} \Phi(z)\right)^{m_{s+1}}=Q_{1, s+1}(z), \\
& \partial_{\mathbf{b}} \Phi(z) Q_{j-1, s}(z)+\partial_{\mathbf{b}} Q_{j, s}(z)= \\
& =\sum_{\substack{n_{1}+2 n_{2}+\ldots+s n_{s}=s \\
0 \leq n_{1} \leq j-2}} c_{j-1, s, n_{1}, \ldots, n_{s}}\left(\partial_{\mathbf{b}} \Phi(z)\right)^{n_{1}+1}\left(\partial_{\mathbf{b}}^{2} \Phi(z)\right)^{n_{2}} \ldots\left(\partial_{\mathbf{b}}^{s} \Phi(z)\right)^{n_{s}}+
\end{aligned}
$$




$$
\begin{gathered}
+\sum_{\substack{n_{1}+2 n_{2}+\ldots+k n_{s}=s \\
0 \leq n_{1} \leq j-1}} c_{j, s, n_{1}, n_{2}, \ldots, n_{s}}\left(n_{1}\left(\partial_{\mathbf{b}} \Phi(z)\right)^{n_{1}-1}\left(\partial_{\mathbf{b}}^{2} \Phi(z)\right)^{n_{2}+1} \ldots\left(\partial_{\mathbf{b}}^{s} \Phi(z)\right)^{n_{s}}+\right. \\
\left.\quad+\ldots+n_{s}\left(\partial_{\mathbf{b}} \Phi(z)\right)^{n_{1}}\left(\partial_{\mathbf{b}}^{2} \Phi(z)\right)^{n_{2}} \ldots\left(\partial_{\mathbf{b}}^{s} \Phi(z)\right)^{n_{s}-1} \partial_{\mathbf{b}}^{s+1} \Phi(z)\right) \\
=\sum_{\substack{m_{1}+2 m_{2}+\ldots+(s+1) m_{s+1}=s+1 \\
0 \leq m_{1} \leq j-1}} \tilde{c}_{j, s+1, m_{1}, . ., m_{s+1}}\left(\partial_{\mathbf{b}} \Phi(z)\right)^{n_{1}} \ldots\left(\partial_{\mathbf{b}}^{s} \Phi(z)\right)^{n_{s}}\left(\partial_{\mathbf{b}}^{s+1} \Phi(z)\right)^{n_{s+1}}=Q_{j, s+1}(z),
\end{gathered}
$$

we obtain (6) with $s+1$ instead of $k$.

Using mathematical induction as in (6) it can be proved that (7) holds. After differentiation in the direction $\mathbf{b}$ equation (7) gives

$$
\begin{aligned}
& \nabla^{s+1} f(\Phi(z), \ldots, \Phi(z))=\frac{\partial_{\mathbf{b}}^{s+1} F(z)}{\left(\partial_{\mathbf{b}} \Phi(z)\right)^{s+1}}-s \partial_{\mathbf{b}}^{2} \Phi(z) \partial_{\mathbf{b}}^{s} F(z)\left(\partial_{\mathbf{b}} \Phi(z)\right)^{-s-2}+ \\
& +\sum_{j=1}^{s-1}\left\{\partial_{\mathbf{b}}^{j+1} F(z)\left(\partial_{\mathbf{b}} \Phi(z)\right)^{j-2 s-1} Q_{j, s}^{*}(z)+\right. \\
& \left.+\partial_{\mathbf{b}}^{j} F(z)\left(\partial_{\mathbf{b}} \Phi(z)\right)^{j-2 s-2}\left((j-2 s) \partial_{\mathbf{b}}^{2} \Phi(z) Q_{j, s}^{*}(z)+\partial_{\mathbf{b}} \Phi(z) \partial_{\mathbf{b}} Q_{j, s}^{*}(z)\right)\right\}= \\
& =\frac{\partial_{\mathbf{b}}^{s+1} F(z)}{\left(\partial_{\mathbf{b}} \Phi(z)\right)^{s+1}}+\partial_{\mathbf{b}}^{s} F(z)\left(\partial_{\mathbf{b}} \Phi(z)\right)^{-s-2}\left(-s \partial_{\mathbf{b}}^{2} \Phi(z)+Q_{s-1, s}^{*}(z)\right)+ \\
& +\sum_{j=2}^{s-1}\left\{\partial_{\mathbf{b}}^{j} F(z)\left(\partial_{\mathbf{b}} \Phi(z)\right)^{j-2 s-2}\left(\partial_{\mathbf{b}} \Phi(z) \partial_{\mathbf{b}} Q_{j, s}^{*}(z)+(j-2 s) \partial_{\mathbf{b}}^{2} \Phi(z) Q_{j, s}^{*}(z)+Q_{j-1, s}^{*}(z)\right)\right\}+ \\
& +\partial_{\mathbf{b}} F(z)\left(\partial_{\mathbf{b}} \Phi(z)\right)^{-2 s-1}\left((1-2 s) \partial_{\mathbf{b}}^{2} \Phi(z) Q_{1, s}^{*}(z)+\partial_{\mathbf{b}} \Phi(z) \partial_{\mathbf{b}} Q_{1, s}^{*}(z)\right) .
\end{aligned}
$$

Since

$$
\begin{aligned}
& -s \partial_{\mathbf{b}}^{2} \Phi(z)+Q_{s-1, s}^{*}(z)=\left(-s+b_{s-1, s, m_{1}, \ldots, m_{s}}\right) \partial_{\mathbf{b}}^{2} \Phi(z)= \\
& =\sum_{\substack{m_{1}+2 m_{2}+\ldots+s m_{s}+\\
+(s+1) m_{s+1}=2}} \tilde{b}_{s, s+1, m_{1}, \ldots, m_{s+1}}\left(\partial_{\mathbf{b}} \Phi(z)\right)^{m_{1}} \ldots\left(\partial_{\mathbf{b}}^{s} F(z)\right)^{m_{s}} \times \\
& \times\left(\partial_{\mathbf{b}}^{m_{s}+1} F(z)\right)^{m_{s}+1}=Q_{s, s+1}^{*}(z), \\
& (1-2 s) \partial_{\mathbf{b}}^{2} \Phi(z) Q_{1, s}^{*}(z)+\partial_{\mathbf{b}} \Phi(z) \partial_{\mathbf{b}} Q_{1, s}^{*}(z)=(1-2 s) \times \\
& \times \sum_{\substack{m_{1}+2 m_{2}+\ldots+s m_{s}=\\
=2 s-2}} b_{1, s, m_{1}, \ldots, m_{s}}\left(\partial_{\mathbf{b}} \Phi(z)\right)^{m_{1}}\left(\partial_{\mathbf{b}}^{2} \Phi(z)\right)^{m_{2}+1} \ldots\left(\partial_{\mathbf{b}}^{s} F(z)\right)^{m_{s}}+ \\
& +\sum_{\substack{m_{1}+2 m_{2}+\ldots+s m_{s}=\\
=2 s-2}} b_{1, s, m_{1}, \ldots, m_{s}}\left\{m_{1}\left(\partial_{\mathbf{b}} \Phi(z)\right)^{m_{1}}\left(\partial_{\mathbf{b}}^{2} \Phi(z)\right)^{m_{2}+1} \ldots\left(\partial_{\mathbf{b}}^{s} F(z)\right)^{m_{s}}+\right. \\
& +m_{2}\left(\partial_{\mathbf{b}} \Phi(z)\right)^{m_{1}+1}\left(\partial_{\mathbf{b}}^{2} \Phi(z)\right)^{m_{2}-1}\left(\partial_{\mathbf{b}}^{3} \Phi(z)\right)^{m_{3}+1} \ldots\left(\partial_{\mathbf{b}}^{s} F(z)\right)^{m_{s}}+\ldots+ \\
& \left.+m_{s}\left(\partial_{\mathbf{b}} \Phi(z)\right)^{m_{1}+1} \ldots\left(\partial_{\mathbf{b}}^{s} F(z)\right)^{m_{s}-1} \partial_{\mathbf{b}}^{s+1} \Phi(z)\right\}= \\
& =\sum_{\substack{m_{1}+2 m_{2}+\ldots+s m_{s}+\\
+(s+1) m_{s+1}=2 s}} \tilde{b}_{1, s+1, m_{1}, \ldots, m_{s+1}}\left(\partial_{\mathbf{b}} \Phi(z)\right)^{m_{1}} \ldots\left(\partial_{\mathbf{b}}^{s} F(z)\right)^{m_{s}}\left(\partial_{\mathbf{b}}^{s+1} \Phi(z)\right)^{m_{s+1}}=Q_{1, s+1}^{*}(z),
\end{aligned}
$$

and

$$
\partial_{\mathbf{b}} \Phi(z) \partial_{\mathbf{b}} Q_{j, s}^{*}(z)+(j-2 s) \partial_{\mathbf{b}}^{2} \Phi(z) Q_{j, s}^{*}(z)+Q_{j-1, s}^{*}(z)=
$$




$$
\begin{aligned}
& =\sum_{\substack{m_{1}+2 m_{2}+\ldots+s m_{s}=\\
=2(s-j)}} b_{j, s, m_{1}, \ldots, m_{s}}\left\{m_{1}\left(\partial_{\mathbf{b}} \Phi(z)\right)^{m_{1}}\left(\partial_{\mathbf{b}}^{2} \Phi(z)\right)^{m_{2}+1} \ldots\left(\partial_{\mathbf{b}}^{s} F(z)\right)^{m_{s}}+\right. \\
& \left.+\ldots+m_{s}\left(\partial_{\mathbf{b}} \Phi(z)\right)^{m_{1}+1}\left(\partial_{\mathbf{b}}^{2} \Phi(z)\right)^{m_{2}} \ldots\left(\partial_{\mathbf{b}}^{s} F(z)\right)^{m_{s}-1} \partial_{\mathbf{b}}^{s+1} \Phi(z)\right\}+ \\
& +(j-2 s) \sum_{\substack{m_{1}+2 m_{2}+\ldots+s m_{s}=\\
=2(s-j)}} b_{j, s, m_{1}, \ldots, m_{s}}\left(\partial_{\mathbf{b}} \Phi(z)\right)^{m_{1}}\left(\partial_{\mathbf{b}}^{2} \Phi(z)\right)^{n_{2}+1} \ldots\left(\partial_{\mathbf{b}}^{s} F(z)\right)^{m_{s}}+ \\
& +\sum_{\substack{m_{1}+2 m_{2}+\ldots+s m_{s}=\\
=2(s-j)+2}} b_{j-1, s, m_{1}, \ldots, m_{s}}\left(\partial_{\mathbf{b}} \Phi(z)\right)^{m_{1}} \ldots\left(\partial_{\mathbf{b}}^{s} F(z)\right)^{m_{s}}= \\
& =\sum_{\substack{m_{1}+2 m_{2}+\ldots+s m_{s}+\\
+(s+1) m_{s+1}=2(s+1-j)}} \tilde{b}_{j, s+1, m_{1}, \ldots, m_{s+1}}\left(\partial_{\mathbf{b}} \Phi(z)\right)^{m_{1}} \ldots\left(\partial_{\mathbf{b}}^{s+1} \Phi(z)\right)^{m_{s+1}}=Q_{j, s+1}^{*}(z),
\end{aligned}
$$

we conclude that (6) is valid with $s+1$ instead of $k$.

Let $f$ be an entire function of bounded $l$-index. By Theorem 3 inequality (5) holds for $n=m, F=f, L=l, \mathbf{b}=\mathbf{1}$. Taking into account (4) and (6), for $k=p+1$ we obtain

$$
\begin{aligned}
& \frac{\left|\partial_{\mathbf{b}}^{p+1} F(z)\right|}{L^{p+1}(z)} \leq \frac{\left|\nabla^{p+1} f(\Phi(z), \ldots, \Phi(z))\right|}{L^{p+1}(z)}\left|\partial_{\mathbf{b}} \Phi(z)\right|^{p+1}+\sum_{j=1}^{p} \frac{\left|\nabla^{j} f(\Phi(z), \ldots, \Phi(z))\right|\left|Q_{j, p+1}(z)\right|}{L^{p+1}(z)} \leq \\
& \leq \max \left\{\frac{\left|\nabla^{k} f(\Phi(z), \ldots, \Phi(z))\right|}{l^{k}(\Phi(z))}: 0 \leq k \leq p\right\}\left(C+\sum_{j=1}^{p} \frac{\left|Q_{j, p+1}(z)\right|}{l^{p+1-j}(\Phi(z))\left|\partial_{\mathbf{b}} \Phi(z)\right|^{p+1}}\right) \leq \\
& \leq \max \left\{\frac{\left|\nabla^{k} f(\Phi(z), \ldots, \Phi(z))\right|}{l^{k}(\Phi(z))}: 0 \leq k \leq p\right\}\left(C+\sum_{j=1}^{p} \sum_{\substack{n_{1}+2 n_{2}+\ldots+(p+1) n_{p+1}=p+1 \\
0 \leq n_{1} \leq j-1}} c_{j, p+1, n_{1}, \ldots, n_{p+1}} \times\right. \\
& \left.\times \frac{\left|\left(\partial_{\mathbf{b}} \Phi(z)\right)^{n_{1}}\left(\partial_{\mathbf{b}}^{2} \Phi(z)\right)^{n_{2}} \ldots\left(\partial_{\mathbf{b}}^{p+1} \Phi(z)\right)^{n_{p+1}}\right|}{l^{p+1-j}(\Phi(z))\left|\partial_{\mathbf{b}} \Phi(z)\right|^{p+1}}\right) \leq \max \left\{\frac{\left|\nabla^{k} f(\Phi(z), \ldots, \Phi(z))\right|}{l^{k}(\Phi(z))}: 0 \leq k \leq p\right\} \times \\
& \times\left(C+\sum_{j=1}^{p} \sum_{\substack{n_{1}+2 n_{2}+\ldots+(p+1) n_{p+1}=p+1 \\
0 \leq n_{1} \leq j-1}} \frac{c_{j, p+1, n_{1}, \ldots, n_{p+1}} K^{p+1}}{l^{p+1-j}(\Phi(z))}\right) \leq C_{1} \max _{0 \leq k \leq p} \frac{\left|\nabla^{k} f(\Phi(z), \ldots, \Phi(z))\right|}{l^{k}(\Phi(z))} .
\end{aligned}
$$

Using (7), we find the upper estimate for the fraction $\frac{\left|\nabla^{k} f(\Phi(z), \ldots, \Phi(z))\right|}{l^{k}(\Phi(z))}$ :

$$
\begin{aligned}
& \frac{\left|\nabla^{k} f(\Phi(z), \ldots, \Phi(z))\right|}{l^{k}(\Phi(z))} \leq \frac{\left|\partial_{\mathbf{b}}^{k} F(z)\right|}{l^{k}(\Phi(z))\left|\partial_{\mathbf{b}} \Phi(z)\right|^{k}}+\sum_{j=1}^{k-1} \frac{\left|\partial_{\mathbf{b}}^{j} F(z)\right|\left|Q_{j, k}^{*}(z)\right|}{l^{k}(\Phi(z))\left|\partial_{\mathbf{b}} \Phi(z)\right|^{2 k-j}} \leq \\
\leq & \max \left\{\frac{1}{L^{j}(z)}\left|\partial_{\mathbf{b}}^{j} F(z)\right|: 1 \leq j \leq k\right\}\left(1+\sum_{j=1}^{k-1} \frac{\left|Q_{j, k}^{*}(z)\right|}{l^{k-j}(\Phi(z))\left|\partial_{\mathbf{b}} \Phi(z)\right|^{2(k-j)}}\right) \leq \\
\leq & \max \left\{\frac{1}{L^{j}(z)}\left|\partial_{\mathbf{b}}^{j} F(z)\right|: 1 \leq j \leq k\right\}\left(1+\sum_{j=1}^{k-1} \sum_{m_{1}+2 m_{2}+\ldots+k m_{k}=2(k-j)}\left|b_{j, k, m_{1}, \ldots, m_{k}}\right| \times\right. \\
& \left.\times \frac{\left|\left(\partial_{\mathbf{b}} \Phi(z)\right)^{m_{1}}\left(\partial_{\mathbf{b}}^{2} \Phi(z)\right)^{m_{2}} \ldots\left(\partial_{\mathbf{b}}^{k} \Phi(z)\right)^{m_{k}}\right|}{l^{k-j}(\Phi(z))\left|\partial_{\mathbf{b}} \Phi(z)\right|^{2(k-j)}}\right) \leq \max \left\{\frac{\left|\partial_{\mathbf{b}}^{j} F(z)\right|}{L^{j}(z)}: 1 \leq j \leq k\right\} \times
\end{aligned}
$$




$$
\times\left(1+\sum_{j=1}^{k-1} \sum_{m_{1}+2 m_{2}+\ldots+k m_{k}=2(k-j)} \frac{\left|b_{j, k, m_{1}, \ldots, m_{k}}\right| K^{k}}{l^{k-j}(\Phi(z))}\right) \leq C_{2} \max _{1 \leq j \leq k} \frac{\left|\partial_{\mathbf{b}}^{j} F(z)\right|}{L^{j}(z)} .
$$

Hence, it follows that

$$
\frac{\left|\partial_{\mathbf{b}}^{p+1} F(z)\right|}{L^{p+1}(z)} \leq C_{1} C_{2} \max \left\{\frac{\left|\partial_{\mathbf{b}}^{k} F(z)\right|}{L^{k}(z)}: 0 \leq k \leq p\right\} .
$$

Therefore, by Theorem 4 the last inequality means that the function $F$ has bounded $L$-index in the direction $\mathbf{b}$.

Conversely, suppose that the function $F$ is of bounded $L$-index in the direction $\mathbf{b}$. Then it satisfies (5). In view of (4) and (7), we deduce

$$
\begin{aligned}
& \frac{\left|\nabla^{p+1} f(\Phi(z), \ldots, \Phi(z))\right|}{l^{p+1}(\Phi(z))} \leq \frac{\left|\partial_{\mathbf{b}}^{p+1} F(z)\right|}{l^{p+1}(\Phi(z))\left|\partial_{\mathbf{b}} \Phi(z)\right|^{p+1}}+\sum_{j=1}^{p} \frac{\left|\partial_{\mathbf{b}}^{j} F(z)\right|\left|Q_{j, p+1}^{*}(z)\right|}{l^{p+1}(\Phi(z))\left|\partial_{\mathbf{b}} \Phi(z)\right|^{2 p+2-j}} \leq \\
& \leq \max \left\{\frac{\left|\partial_{\mathbf{b}}^{k} F(z)\right|}{L^{k}(z)}: 0 \leq k \leq p\right\}\left(C+\sum_{j=1}^{p} \frac{\left|Q_{j, p+1}^{*}(z)\right|}{l^{p+1-j}(\Phi(z))\left|\partial_{\mathbf{b}} \Phi(z)\right|^{2(p+1-j)}}\right) \leq \\
& \leq \max \left\{\frac{\left|\partial_{\mathbf{b}}^{k} F(z)\right|}{L^{k}(z)}: 0 \leq k \leq p\right\}\left(C+\sum_{j=1}^{p} \sum_{\substack{m_{1}+\ldots+(p+1) m_{p+1}=\\
=2(p+1-j)}}\left|b_{j, p+1, m_{1}, \ldots, m_{p+1}}\right| \times\right. \\
& \left.\times \frac{\left|\left(\partial_{\mathbf{b}} \Phi(z)\right)^{m_{1}}\left(\partial_{\mathbf{b}}^{2} \Phi(z)\right)^{m_{2}} \ldots\left(\partial_{\mathbf{b}}^{p+1} \Phi(z)\right)^{m_{p+1}}\right|}{l^{p+1-j}(\Phi(z))\left|\partial_{\mathbf{b}} \Phi(z)\right|^{2(p+1-j)}}\right) \leq \max \left\{\frac{\left|\partial_{\mathbf{b}}^{k} F(z)\right|}{L^{k}(z)}: 0 \leq k \leq p\right\} \times \\
& \times\left(C+\sum_{j=1}^{p} \sum_{\substack{m_{1}+\ldots+(p+1) m_{p+1}=\\
=2(p+1-j)}} \frac{\left|b_{j, p+1, m_{1}, \ldots, m_{p+1}}\right| K^{2 p+2-2 j}}{l^{p+1-j}(\Phi(z))}\right) \leq C_{3} \max _{0 \leq k \leq p} \frac{\left|\partial_{\mathbf{b}}^{k} F(z)\right|}{L^{k}(z)} .
\end{aligned}
$$

Applying (6), we estimate

$$
\begin{gathered}
\frac{\left|\partial_{\mathbf{b}}^{k} F(z)\right|}{L^{k}(z)} \leq \frac{\left|\nabla^{k} f(\Phi(z), \ldots, \Phi(z))\right|\left|\varphi^{\prime}(z)\right|^{k}}{L^{k}(z)}+\sum_{j=1}^{k-1} \frac{\left|\nabla^{j} f(\Phi(z), \ldots, \Phi(z))\right|\left|Q_{j, k}(z)\right|}{L^{k}(z)} \leq \\
\leq \max \left\{\frac{\left|\nabla^{j} f(\Phi(z), \ldots, \Phi(z))\right|}{l^{j}(\Phi(z))}: 1 \leq j \leq k\right\}\left(1+\sum_{j=1}^{k-1} \frac{\left|Q_{j, k}(z)\right|}{l^{k-j}(\Phi(z))\left|\partial_{\mathbf{b}} \Phi(z)\right|^{k}}\right) \leq \\
\leq C_{4} \max \left\{\frac{\left|\nabla^{j} f(\Phi(z), \ldots, \Phi(z))\right|}{l^{j}(\Phi(z))}: 1 \leq j \leq k\right\} .
\end{gathered}
$$

It implies that

$$
\frac{\left|\nabla^{p+1} f(\Phi(z), \ldots, \Phi(z))\right|}{l^{p+1}(\Phi(z))} \leq C_{3} C_{4} \max \left\{\frac{\left|\nabla^{j} f(\Phi(z), \ldots, \Phi(z))\right|}{l^{j}(\Phi(z))}: 0 \leq j \leq p\right\} .
$$

Thus, by Theorem $3(n=m, F=f, L=l, \mathbf{b}=\mathbf{1})$ the function $f$ has bounded $l$-index. 
Note that the condition $\partial_{\mathbf{b}} \Phi(z) \neq 0$ in Theorem 1 is generated by our method of the proof. In fact, we can remove it and prove more general proposition with some greater function $L$.

Theorem 5. Let $\mathbf{b} \in \mathbb{C}^{n} \backslash\{\mathbf{0}\}, f: \mathbb{C}^{m} \rightarrow \mathbb{C}$ be an entire function, $\Phi: \mathbb{B}^{n} \rightarrow \mathbb{C}$ be an analytic function, $p=N_{\mathbf{1}}(f, l)$ or $p=N_{\mathbf{b}}(F, L)$ respective.

Suppose that $l \in Q_{1}^{m}, l(w) \geq 1, w \in \mathbb{C}^{m}$ and $L \in Q_{\mathbf{b}}\left(\mathbb{B}^{n}\right)$ with

$$
L(z)=\max _{1 \leq j \leq p}\left\{1,\left|\partial_{\mathbf{b}}^{j} \Phi(z)\right|\right\} l(\underbrace{\Phi(z), \ldots, \Phi(z)}_{m \text { times }}) .
$$

The entire function $f$ has bounded l-index in the direction $\mathbf{1}$ if and only if the analytic function $F(z)=f(\underbrace{\Phi(z), \ldots, \Phi(z)}_{m \text { times }})$ has bounded L-index in the direction $\mathbf{b}$.

The proof of this theorem is similar to that of Theorem 2 and also use analogs of Hayman's Theorem for entire functions of bounded $L$-index in direction (Theorems 3,4 ).

Remark 1. One should observe that Theorems 2 and 5 are also new results in one-dimensional case, i.e. in the case of analytic functions in the unit disc. Moreover, if we replace the condition " $\Phi$ be an analytic function in the unit ball" by the condition " $\Phi$ be an entire function of several variables" in these theorems then we also deduce new results for composite entire functions. In comparison, there is removed the condition $\partial_{\mathbf{b}} \Phi(z) \neq 0$ and is considered more general composition than in [16].

Note that for $n=1$ the assumption in Theorem 2 are weaker than in [19] because we require validity of (4) for $j \leq p$ instead all values $j \in \mathbb{N}$.

3. Product theorem. To prove a theorem on product of analytic functions of bounded $L$-index in direction we need auxiliary propositions.

Lemma 1 ([5,6]). Let $L \in Q_{\mathbf{b}}\left(\mathbb{B}^{n}\right), \frac{1}{\beta}<\theta_{1} \leq \theta_{2}<+\infty, \theta_{1} L(z) \leq L^{*}(z) \leq \theta_{2} L(z)$. Analytic function $F(z)$ in $\mathbb{B}^{n}$ has bounded $L^{*}$-index in the direction $\mathbf{b}$ if and only if the function $F$ has bounded $L$-index in the direction $\mathbf{b}$.

Let $g_{z^{0}}(t):=F\left(z^{0}+t \mathbf{b}\right)$. If for given $z^{0} \in \mathbb{B}^{n} \quad g_{z^{0}}(t) \neq 0$ for all $t \in D_{z^{0}}$, then $G_{r}^{\mathbf{b}}\left(F, z^{0}\right):=$ $\varnothing$; if for given $z^{0} \in \mathbb{B}^{n} \quad g_{z^{0}}(t) \equiv 0$, then $G_{r}^{\mathbf{b}}\left(F, z^{0}\right):=\left\{z^{0}+t \mathbf{b}: t \in D_{z^{0}}\right\}$. And if for some $z^{0} \in \mathbb{B}^{n} \quad g_{z^{0}}(t) \not \equiv 0$ and $a_{k}^{0}$ are zeros of the functions $g_{z^{0}}(t)$, i.e., $F\left(z^{0}+a_{k}^{0} \mathbf{b}\right)=0$, then

$$
G_{r}^{\mathbf{b}}\left(F, z^{0}\right):=\bigcup_{k}\left\{z^{0}+t \mathbf{b}:\left|t-a_{k}^{0}\right| \leq \frac{r}{L\left(z^{0}+a_{k}^{0} \mathbf{b}\right)}\right\}, \quad r>0 .
$$

Let

$$
G_{r}^{\mathbf{b}}(F)=\bigcup_{z^{0} \in \mathbb{B}^{n}} G_{r}^{\mathbf{b}}\left(F, z^{0}\right)
$$

By $n\left(r, z^{0}, 1 / F\right)=\sum_{\left|a_{k}^{0}\right| \leq r} 1$ we denote counting functions of zeros $a_{k}^{0}$.

Theorem $6([5,6])$. Let $F$ be an analytic function in $\mathbb{B}^{n}, L \in Q_{\mathbf{b}}\left(\mathbb{B}^{n}\right)$ and $\mathbb{B}^{n} \backslash G_{\beta}^{\mathbf{b}}(F) \neq \varnothing$. The function $F(z)$ has bounded $L$-index in the direction $\mathbf{b}$ if and only if

1) for every $r \in(0, \beta]$ there exists $P=P(r)>0$ such that for any $z \in \mathbb{B}^{n} \backslash G_{r}^{\mathbf{b}}(F)$

$$
\left|\frac{\partial_{\mathbf{b}} F(z)}{F(z)}\right| \leq P L(z)
$$


2) for each $r \in(0, \beta]$ there exists $\widetilde{n}(r) \in \mathbb{Z}_{+}$such that for all $z^{0} \in \mathbb{B}^{n}$ with $F\left(z^{0}+t \mathbf{b}\right) \not \equiv 0$ one has

$$
n\left(\frac{r}{L\left(z^{0}\right)}, z^{0}, \frac{1}{F}\right) \leq \widetilde{n}(r)
$$

Using Theorem 4 we prove the following

Theorem 7. Let $L \in Q_{\mathbf{b}}\left(\mathbb{B}^{n}\right)$. An analytic function $F: \mathbb{B}^{n} \rightarrow \mathbb{C}$ has bounded $L$-index in the direction $\mathbf{b}$ if and only if there exist numbers $C \in(0,+\infty)$ and $N \in \mathbb{N}$ such that for all $z \in \mathbb{B}^{n}$

$$
\sum_{k=0}^{N} \frac{\left|\partial_{\mathbf{b}}^{k} F(z)\right|}{k ! L^{k}(z)} \geq C \sum_{k=N+1}^{\infty} \frac{\left|\partial_{\mathbf{b}}^{k} F(z)\right|}{k ! L^{k}(z)}
$$

Proof. Proof of this theorem is similar to the proof of its analogs for entire functions of bounded $L$-index in direction [8] and for entire functions of bounded $l$-index [23].

Let $\frac{1}{\beta}<\theta<1$. If the function $F$ is of bounded $L$-index in the direction $\mathbf{b}$, then by Lemma $1 F$ is also of bounded $L^{*}$-index in the direction $\mathbf{b}$, where $L^{*}(z)=\theta L(z)$. Denote $N^{*}=N_{\mathbf{b}}\left(F, L_{*}\right)$ and $N=N_{\mathbf{b}}(F, L)$. Thus,

$$
\begin{aligned}
& \max \left\{\frac{\left|\partial_{\mathbf{b}}^{k} F(z)\right|}{k ! L^{k}(z)}: 0 \leq k \leq N^{*}\right\}=\max \left\{\frac{\left|\partial_{\mathbf{b}}^{k} F(z)\right|}{k ! L_{*}^{k}(z)} \theta^{k}: 0 \leq k \leq N^{*}\right\} \geq \\
& \geq \theta^{N^{*}} \max \left\{\frac{\left|\partial_{\mathbf{b}}^{k} F(z)\right|}{k ! L_{*}^{k}(z)}: 0 \leq k \leq N^{*}\right\} \geq \theta^{N^{*}} \frac{\left|\partial_{\mathbf{b}}^{j} F(z)\right|}{j ! L_{*}^{j}(z)}=\theta^{N^{*}-j} \frac{\left|\partial_{\mathbf{b}}^{j} F(z)\right|}{j ! L^{j}(z)}
\end{aligned}
$$

for all $j \geq 0$ and

$$
\begin{aligned}
& \sum_{j=N^{*}+1}^{\infty} \frac{\left|\partial_{\mathbf{b}}^{j} F(z)\right|}{j ! L^{j}(z)} \leq \max \left\{\frac{\left|\partial_{\mathbf{b}}^{k} F(z)\right|}{k ! L^{k}(z)}: 0 \leq k \leq N^{*}\right\} \sum_{j=N^{*}+1}^{\infty} \theta^{j-N^{*}}= \\
& =\frac{\theta}{1-\theta} \max \left\{\frac{\left|\partial_{\mathbf{b}}^{k} F(z)\right|}{k ! L^{k}(z)}: 0 \leq k \leq N^{*}\right\} \leq \frac{\theta}{1-\theta} \sum_{k=0}^{N^{*}} \frac{\left|\partial_{\mathbf{b}}^{k} F(z)\right|}{k ! L^{k}(z)},
\end{aligned}
$$

i.e. we obtain (11) with $N=N^{*}$ and $C=\frac{1-\theta}{\theta}$.

Now we prove the sufficiency. From (11) we obtain

$$
\frac{\left|\partial_{\mathbf{b}}^{N+1} F(z)\right|}{(N+1) ! L^{N+1}(z)} \leq \sum_{k=N+1}^{\infty} \frac{\left|\partial_{\mathbf{b}}^{k} F(z)\right|}{k ! L^{k}(z)} \leq \frac{1}{C} \sum_{k=0}^{N} \frac{\left|\partial_{\mathbf{b}}^{k} F(z)\right|}{k ! L^{k}(z)} \leq \frac{N+1}{C} \max \left\{\frac{\left|\partial_{\mathbf{b}}^{k} F(z)\right|}{k ! L^{k}(z)}: 0 \leq k \leq N\right\} .
$$

Applying Theorem 4, we obtain a desired conclusion.

We then consider an application of Theorem 6 .

Theorem 8. Let $L \in Q_{\mathbf{b}}\left(\mathbb{B}^{n}\right), F: \mathbb{B}^{n} \rightarrow \mathbb{C}$ be an analytic function of bounded $L$-index in the direction $\mathbf{b} \in \mathbb{C}^{n} \backslash\{\mathbf{0}\}, \Phi: \mathbb{B}^{n} \rightarrow \mathbb{C}$ be an analytic function in the unit ball and $\Psi(z)=F(z) \Phi(z)$. The function $\Psi(z)$ is of bounded $L$-index in the direction $\mathbf{b}$ if and only if the function $\Phi(z)$ is of bounded $L$-index in the direction $\mathbf{b}$. 
Proof. The similar result was obtained for entire functions of bounded $L$-index in direction in [8]. Our proof is similar to the proof for entire functions in [8] but now we use Theorem 6 , deduced for functions analytic in the unit ball. Since an analytic function $F(z)$ has bounded $L$-index in the direction $\mathbf{b}$, by Theorem 6 for every $r \in(0, \beta)$ there exists $\widetilde{n}(r) \in \mathbb{Z}_{+}$such that for all $z^{0} \in \mathbb{B}^{n}$, satisfying $F\left(z^{0}+t \mathbf{b}\right) \not \equiv 0$, the estimate $n\left(\frac{r}{L\left(z^{0}\right)}, z^{0}, \frac{1}{F}\right) \leq \widetilde{n}(r)$ holds. Hence,

$$
n\left(\frac{r}{L\left(z^{0}\right)}, z^{0}, \frac{1}{\Phi}\right) \leq n\left(\frac{r}{L\left(z^{0}\right)}, z^{0}, \frac{1}{\Psi}\right) \leq n\left(\frac{r}{L\left(z^{0}\right)}, z^{0}, \frac{1}{\Phi}\right)+\widetilde{n}(r) .
$$

Thus, condition 2 of Theorem 6 either holds or does not hold for functions $\Psi(z)$ and $\Phi(z)$ simultaneously. If $\Phi(z)$ has bounded $L$-index in the direction $\mathbf{b}$, then for every $r \in(0, \beta)$ there exist numbers $P_{F}(r)>0$ and $P_{\Phi}(r)>0$ such that $\left|\frac{\partial_{\mathbf{b}} F(z)}{F(z)}\right| \leq P_{f}(r) L(z),\left|\frac{\partial_{\mathbf{b}} \Phi(z)}{\Phi(z)}\right| \leq P_{\Phi}(r) L(z)$ for each $z \in\left(\mathbb{B}^{n} \backslash G_{r}^{\mathbf{b}}(F)\right) \cap\left(\mathbb{B}^{n} \backslash G_{r}^{\mathbf{b}}(\Phi)\right)$. Since

$$
\mathbb{B}^{n} \backslash G_{r}^{\mathbf{b}}(\Psi) \subset\left(\mathbb{B}^{n} \backslash G_{r}^{\mathbf{b}}(F)\right) \cap\left(\mathbb{B}^{n} \backslash G_{r}^{\mathbf{b}}(\Phi)\right), \quad\left|\frac{\partial_{\mathbf{b}} \Psi(z)}{\Psi(z)}\right| \leq\left|\frac{\partial_{\mathbf{b}} F(z)}{F(z)}\right|+\left|\frac{\partial_{\mathbf{b}} \Phi(z)}{\Phi(z)}\right|,
$$

for all $z \in \mathbb{B}^{n} \backslash G_{r}^{\mathbf{b}}(\Psi)$ we have $\left|\frac{\partial_{\mathbf{b}} \Psi(z)}{\Psi(z)}\right| \leq\left(P_{F}(r)+P_{\Phi}(r)\right) L(z)$, i.e. by Theorem 6 the function $\Psi(z)$ is of bounded $L$-index in the direction $\mathbf{b}$.

On the contrary, let $\Psi(z)$ be of bounded $L$-index in the direction $\mathbf{b}, r>0$. At first we show that for every $z^{0} \in \mathbb{B}^{n} \backslash G_{r}^{\mathbf{b}}(F)(r>0)$ and for every $\widetilde{d}^{k}=z^{0}+d_{k}^{0} \mathbf{b}$, where $d_{k}^{0}$ are zeros of function $\Phi\left(z^{0}+t \mathbf{b}\right)$, we have

$$
\left|z^{0}-\widetilde{d}^{k}\right|>\frac{r|\mathbf{b}|}{2 L\left(z^{0}\right) \lambda_{\mathbf{b}}(r)}
$$

On the other hand, let there exist $z^{0} \in \mathbb{B}^{n} \backslash G_{r}^{\mathbf{b}}(\Phi)$ and $\widetilde{d^{k}}=z^{0}+d_{k}^{0} \mathbf{b}$ such that $\left|z^{0}-\widetilde{d^{k}}\right| \leq$ $\frac{r|\mathbf{b}|}{2 L\left(z^{0}\right) \lambda_{\mathbf{b}}(r)}$. Then by the definition of $\lambda_{\mathbf{b}}$ we have the next estimate $L\left(\widetilde{d^{k}}\right) \leq \lambda_{\mathbf{b}}(r) L\left(z^{0}\right)$. Hence $\left|z^{0}-\widetilde{d}^{k}\right|=|\mathbf{b}| \cdot\left|d_{k}^{0}\right| \leq \frac{r|\mathbf{b}|}{2 L\left(\widetilde{d}^{k}\right)}$, i.e. $\left|d_{k}^{0}\right| \leq \frac{r}{2 L\left(\widetilde{d}^{k}\right)}$, but it contradicts $z^{0} \in \mathbb{B}^{n} \backslash G_{r}^{\mathbf{b}}(\Phi)$.

We consider

$$
\bar{K}_{0}=\left\{z^{0}+t \mathbf{b}:|t| \leq \frac{r}{2 L\left(z^{0}\right) \lambda_{\mathbf{b}}(r)}\right\} .
$$

It does not contain zeros of $\Phi\left(z^{0}+t \mathbf{b}\right)$, which may contain zeros $\widetilde{c}^{k}=z^{0}+c_{k}^{0} \mathbf{b}$ of the function $\Psi\left(z^{0}+t \mathbf{b}\right)$. Since $\Psi(z)$ is of bounded $L$-index in the direction $\mathbf{b}$, the set $\bar{K}_{0}$ by Theorem 6 contains at most $\widetilde{n}_{1}=\widetilde{n_{1}}\left(\frac{r}{2 \lambda_{\mathbf{b}}(r)}\right)$ zeros $c_{k}^{0}$ of the function $\Psi\left(z^{0}+t \mathbf{b}\right)$. For all $c_{k}^{0} \in \bar{K}_{0}$, using the definition of $Q_{\mathbf{b}}\left(\mathbb{B}^{n}\right)$, we obtain the following inequality

$$
L\left(z^{0}+c_{k}^{0} \mathbf{b}\right) \geq \frac{1}{\lambda_{\mathbf{b}}\left(\frac{r}{\lambda_{\mathbf{b}}(r)}\right)} L\left(z^{0}\right) .
$$

Thus, every set $m_{k}^{0}=\left\{z^{0}+t \mathbf{b}:\left|t-c_{k}^{0}\right| \leq \frac{r_{1}}{L\left(z^{0}+c_{k}^{0} \mathbf{b}\right)}\right\}$ with $r_{1}=\frac{r}{4\left(\widetilde{n}_{1}+1\right) \lambda_{\mathbf{b}}\left(\frac{r}{\lambda_{\mathbf{b}}(r)}\right) \lambda_{\mathbf{b}}(r)}$ is contained in the set

$$
s_{k}^{0}=\left\{z^{0}+t \mathbf{b}:\left|t-c_{k}^{0}\right| \leq \frac{r_{1} \lambda_{\mathbf{b}}\left(\frac{r}{\lambda_{\mathbf{b}}(r)}\right)}{L\left(z^{0}\right)}\right\} \text {. }
$$


The total sum of diameters of these sets does not exceed

$$
\frac{2 \widetilde{n}_{1} r_{1} \lambda_{\mathbf{b}}\left(\frac{r}{\lambda_{\mathbf{b}}(r)}\right)}{L\left(z^{0}\right)}=\frac{r}{2 \lambda_{\mathbf{b}}(r) L\left(z^{0}\right)} \cdot \frac{\widetilde{n}_{1}}{\left(\widetilde{n}_{1}+1\right)}<\frac{r}{2 \lambda_{\mathbf{b}}(r) L\left(z^{0}\right)} .
$$

Therefore, there exists $r^{*} \in\left(0, \frac{r}{2 \lambda_{\mathbf{b}}(r)}\right)$ such that if $|t|=\frac{r^{*}}{L\left(z^{0}\right)}$, then $z^{0}+t \mathbf{b} \notin G_{r_{1}}^{\mathbf{b}}(\Psi)$, and therefore $z^{0}+t \mathbf{b} \notin G_{r_{1}}^{\mathbf{b}}(F)$. By Theorem 6 for all these points $z^{0}+t \mathbf{b}$ we obtain

$$
\left|\frac{\partial_{\mathbf{b}} \Phi\left(z^{0}+t \mathbf{b}\right)}{\Phi\left(z^{0}+t \mathbf{b}\right)}\right| \leq\left|\frac{\partial_{\mathbf{b}} \Psi\left(z^{0}+t \mathbf{b}\right)}{\Psi\left(z^{0}+t \mathbf{b}\right)}\right|+\left|\frac{\partial_{\mathbf{b}} F\left(z^{0}+t \mathbf{b}\right)}{F\left(z^{0}+t \mathbf{b}\right)}\right| \leq\left(P_{\Psi}^{*}+P_{F}^{*}\right) L\left(z^{0}+t \mathbf{b}\right),
$$

where $P_{\Psi}^{*}$ and $P_{F}^{*}$ depend only on $r_{1}$, i.e. only on $r$. Since the function $\frac{\partial_{\mathbf{b}} \Phi(z)}{\Phi(z)}$ is analytic in $\bar{K}_{0}$, applying the maximum modulus principle to the function $\frac{\partial_{\mathbf{b}} \Phi\left(z^{0}+t \mathbf{b}\right)}{\Phi\left(z^{0}+t \mathbf{b}\right)}$ as a function of variable $t$, we obtain that the modulus of this function at the point $t=0$ does not exceed the maximum modulus of this function on the circle $\left\{t \in \mathbb{C}:|t|=\frac{r^{*}}{L\left(z^{0}\right)}\right\}$. It means that obtained inequality (13) holds for $z^{0}$.

Thus, for arbitrary $r \in(0, \beta)$ and $z^{0} \in \mathbb{B}^{n} \backslash G_{r}^{\mathbf{b}}(F)$ we have proved the first condition of Theorem 6. Above we have already shown that the second condition of Theorem 6 is true. Hence, by the mentioned theorem the function $\Phi(z)$ has bounded $L$-index in the direction $\mathbf{b}$.

4. Boundedness of $L$-index in direction for sum of analytic functions. There are known sufficient conditions of index boundedness for sum of two entire functions of one variables [26]. These results were generalized for entire functions of bounded $L$-index in direction [1] and for entire functions of bounded index in joint variables [3]. But similar conditions for analytic functions in the unit ball (or in the unit disk) are not known. Therefore, in this subsection we consider the following question: what are sufficient conditions for $L$-index boundedness in direction for the sum of two functions analytic in the unit ball?

We need the following theorem.

Theorem 9 ([5,6]). Let $\beta>1, L \in Q_{\mathbf{b}, \beta}\left(\mathbb{B}^{n}\right)$. An analytic function $F(z)$ in $\mathbb{B}^{n}$ has bounded $L$-index in the direction $\mathbf{b} \in \mathbb{C}^{n} \backslash\{\mathbf{0}\}$ if and only if for any $r_{1}$ and for any $r_{2}, 0<r_{1}<r_{2} \leq \beta$, there exists $P_{1}=P_{1}\left(r_{1}, r_{2}\right) \geq 1$ such that for each $z^{0} \in \mathbb{B}^{n}$

$$
\max \left\{\left|F\left(z^{0}+t \mathbf{b}\right)\right|:|t|=\frac{r_{2}}{L\left(z^{0}\right)}\right\} \leq P_{1} \max \left\{\left|F\left(z^{0}+t \mathbf{b}\right)\right|:|t|=\frac{r_{1}}{L\left(z_{0}\right)}\right\} .
$$

Let us consider intersection of the hyperplane $\langle z, \mathbf{b}\rangle=0$ with the unit ball. The intersection we denote by $A=\left\{z \in \mathbb{B}^{n}:\langle z, \mathbf{b}\rangle=0\right\}$, where $\langle z, \mathbf{b}\rangle:=\sum_{j=1}^{n} z_{j} b_{j}$. Obviously $\bigcup_{z^{0} \in A}\left\{z^{0}+t \mathbf{b}:|t| \leq \frac{1-\left|z_{0}\right|}{|\mathbf{b}|}\right\}=\mathbb{B}^{n}$.

Let $z^{0} \in A$ be a given point. If $F\left(z^{0}+t \mathbf{b}\right) \not \equiv 0$ as a function of variable $t \in \mathbb{C}$, then there exists $t_{0} \in D_{z^{0}}$ such that $F\left(z^{0}+t_{0} \mathbf{b}\right) \neq 0$. We denote

$$
\begin{gathered}
B\left(z^{0}, t\right)=\left\{t_{0} \in D_{z^{0}}:\left|t_{0}-t\right|<\min \left\{\frac{\beta}{2 L\left(z^{0}+t \mathbf{b}\right)}, \frac{1-\left|z^{0}+\mathbf{b} t\right|}{2|\mathbf{b}|}\right\}, F\left(z^{0}+t_{0} \mathbf{b}\right) \neq 0\right\}, \\
B\left(z^{0}\right)=\bigcup_{|t| \leq\left(1-\left|z^{0}\right|\right) / \mathbf{b} \mid} B\left(z^{0}, t\right) .
\end{gathered}
$$


Theorem 10. Let $L: \mathbb{B}^{n} \rightarrow \mathbb{R}_{+}$be a positive continuous function satisfying (2) with $\beta \geq 3$, and $F: \mathbb{B}^{n} \rightarrow \mathbb{R}_{+}, G: \mathbb{B}^{n} \rightarrow \mathbb{R}_{+}$be analytic functions in the unit ball which obey the following conditions:

1) $G(z)$ has bounded $L$-index in the direction $\mathbf{b} \in \mathbb{C}^{n} \backslash\{\mathbf{0}\}$ with $N_{b}(G, L)=N<+\infty$;

2) there exists $\alpha \in(0,1)$ such that for all $z \in \mathbb{B}^{n}$ and $p \geq N+1(p \in \mathbb{N})$

$$
\frac{\left|\partial_{\mathbf{b}}^{p} G(z)\right|}{p ! L^{p}(z)} \leq \alpha \max \left\{\frac{\left|\partial_{\mathbf{b}}^{k} G(z)\right|}{k ! L^{k}(z)}: 0 \leq k \leq N\right\}
$$

3) for every $z=z^{0}+t \mathbf{b} \in \mathbb{B}^{n}$ with $z^{0} \in A$ and some $t_{0} \in B\left(z^{0}, t\right)$ with $r=\left|t-t_{0}\right| L\left(z^{0}+t \mathbf{b}\right)$ the inequality

$$
\max \left\{\left|F\left(z^{0}+t^{\prime} \mathbf{b}\right)\right|:\left|t^{\prime}-t_{0}\right|=\frac{2 r}{L\left(z^{0}+t \mathbf{b}\right)}\right\} \leq \max \left\{\frac{\left|\partial_{\mathbf{b}}^{k} G\left(z^{0}+t \mathbf{b}\right)\right|}{k ! L^{k}\left(z^{0}+t \mathbf{b}\right)}: 0 \leq k \leq N\right\} ;
$$

is valid;

4) either $(\exists c>0)\left(\forall z^{0} \in A\right)\left(\forall t \in \mathbb{D}_{z^{0}}\right)\left(\exists t_{0} \in B\left(z^{0}, t\right)\right.$ obeying (16) and if $\left|t-t_{0}\right| L\left(z^{0}+\right.$ tb) $\leq 1)$ then

$$
\max \left\{\left|F\left(z^{0}+t^{\prime} \mathbf{b}\right)\right|:\left|t^{\prime}-t_{0}\right|=\frac{2}{L\left(z^{0}+t \mathbf{b}\right)}\right\} /\left|F\left(z^{0}+t_{0} \mathbf{b}\right)\right| \leq c<+\infty,
$$

or for $L \in Q_{\mathbf{b}}\left(\mathbb{B}^{n}\right) \quad(\exists c>0)\left(\forall z^{0} \in A\right)\left(\exists t_{0} \in B\left(z^{0}\right)\right)$ such that (16) is true and

$$
\max \left\{\left|F\left(z^{0}+t^{\prime} \mathbf{b}\right)\right|:\left|t^{\prime}-t_{0}\right|=\frac{2 \lambda_{\mathbf{b}}(1)}{L\left(z^{0}+t_{0} \mathbf{b}\right)}\right\} /\left|F\left(z^{0}+t_{0} \mathbf{b}\right)\right| \leq c<+\infty,
$$

where $\beta \geq 2 \lambda_{\mathbf{b}}(1)$.

Then for every $\varepsilon \in \mathbb{C},|\varepsilon| \leq \frac{1-\alpha}{2 c}$, the function

$$
H(z)=G(z)+\varepsilon F(z)
$$

has bounded $L$-index in the direction $\mathbf{b}$ and $N_{\mathbf{b}}(H, L) \leq N$.

Proof. We write Cauchy's formula for the analytic function $F\left(z^{0}+t \mathbf{b}\right)$ as function of one complex variable $t$

$$
\frac{\partial_{\mathbf{b}}^{p} F\left(z^{0}+t \mathbf{b}\right)}{p !}=\frac{1}{2 \pi i} \int_{\left|t^{\prime}-t\right|=\frac{r}{L\left(z^{0}+t \mathbf{b}\right)}} \frac{F\left(z^{0}+t^{\prime} \mathbf{b}\right)}{\left(t^{\prime}-t\right)^{p+1}} d t^{\prime} .
$$

For the chosen $r=\left|t-t_{0}\right| L\left(z^{0}+t \mathbf{b}\right)$ we deduce

$$
\frac{r}{L\left(z^{0}+t \mathbf{b}\right)}=\left|t^{\prime}-t\right| \geq\left|t^{\prime}-t_{0}\right|-\left|t-t_{0}\right|=\left|t^{\prime}-t_{0}\right|-\frac{r}{L\left(z^{0}+t \mathbf{b}\right)} .
$$

Hence,

$$
\left|t^{\prime}-t_{0}\right| \leq \frac{2 r}{L\left(z^{0}+t \mathbf{b}\right)}
$$


Equality (19) yields

$$
\begin{gathered}
\frac{\left|\partial_{\mathbf{b}}^{p} F\left(z^{0}+t \mathbf{b}\right)\right|}{p ! L^{p}\left(z^{0}+t \mathbf{b}\right)} \leq \frac{1}{2 \pi L^{p}\left(z^{0}+t \mathbf{b}\right)} \cdot \frac{L^{p+1}\left(z^{0}+t \mathbf{b}\right)}{r^{p+1}} \times \\
\times \frac{2 \pi r}{L\left(z^{0}+t \mathbf{b}\right)} \cdot \max \left\{\left|F\left(z^{0}+t^{\prime} \mathbf{b}\right)\right|:\left|t^{\prime}-t\right|=\frac{r}{L\left(z^{0}+t \mathbf{b}\right)}\right\} \leq \\
\leq \frac{1}{r^{p}} \max \left\{\left|F\left(z^{0}+t^{\prime} \mathbf{b}\right)\right|:\left|t^{\prime}-t_{0}\right|=\frac{2 r}{L\left(z^{0}+t \mathbf{b}\right)}\right\} .
\end{gathered}
$$

If $r=\left|t-t_{0}\right| L\left(z^{0}+t \mathbf{b}\right)>1$, then (21) yields

$$
\frac{\left|\partial_{\mathbf{b}}^{p} F\left(z^{0}+t \mathbf{b}\right)\right|}{p ! L^{p}\left(z^{0}+t \mathbf{b}\right)} \leq \max \left\{\left|F\left(z^{0}+t^{\prime} \mathbf{b}\right)\right|:\left|t^{\prime}-t_{0}\right|=\frac{2 r}{L\left(z^{0}+t \mathbf{b}\right)}\right\} .
$$

Let $r=\left|t-t_{0}\right| L\left(z^{0}+t \mathbf{b}\right) \in(0 ; 1]$. Setting $r=1$ in (19) and (20), we analogously deduce

$$
\begin{gathered}
\frac{\left|\partial_{\mathbf{b}}^{p} F\left(z^{0}+t \mathbf{b}\right)\right|}{p ! L^{p}\left(z^{0}+t \mathbf{b}\right)} \leq \max \left\{\left|F\left(z^{0}+t^{\prime} \mathbf{b}\right)\right|:\left|t^{\prime}-t_{0}\right|=\frac{2}{L\left(z^{0}+t \mathbf{b}\right)}\right\}= \\
\quad=\frac{\max \left\{\left|F\left(z^{0}+t^{\prime} \mathbf{b}\right)\right|:\left|t^{\prime}-t_{0}\right|=\frac{2}{L\left(z^{0}+t \mathbf{b}\right)}\right\}}{\max \left\{\left|F\left(z^{0}+t^{\prime} \mathbf{b}\right)\right|:\left|t^{\prime}-t_{0}\right|=\frac{2 r}{L\left(z^{0}+t \mathbf{b}\right)}\right\}} \times \\
\quad \times \max \left\{\left|F\left(z^{0}+t^{\prime} \mathbf{b}\right)\right|:\left|t^{\prime}-t_{0}\right|=\frac{2 r}{L\left(z^{0}+t \mathbf{b}\right)}\right\} \leq \\
\quad \leq \frac{\max \left\{\left|F\left(z^{0}+t^{\prime} \mathbf{b}\right)\right|:\left|t^{\prime}-t_{0}\right|=\frac{2}{L\left(z^{0}+t \mathbf{b}\right)}\right\}}{\left|F\left(z^{0}+t_{0} \mathbf{b}\right)\right|} \times \\
\quad \times \max \left\{\left|F\left(z^{0}+t^{\prime} \mathbf{b}\right)\right|:\left|t^{\prime}-t_{0}\right|=\frac{2 r}{L\left(z^{0}+t \mathbf{b}\right)}\right\} \leq \\
\leq c \max \left\{\left|F\left(z^{0}+t^{\prime} \mathbf{b}\right)\right|:\left|t^{\prime}-t_{0}\right|=\frac{2 r}{L\left(z^{0}+t \mathbf{b}\right)}\right\},
\end{gathered}
$$

where

$$
c=\sup _{z^{0} \in A,|t|<\left(1-\left|z^{0}\right|\right) /|\mathbf{b}|} \frac{\max \left\{\left|F\left(z^{0}+t^{\prime} \mathbf{b}\right)\right|:\left|t^{\prime}-t_{0}\right|=\frac{2}{L\left(z^{0}+t \mathbf{b}\right)}\right\}}{\left|F\left(z^{0}+t_{0} \mathbf{b}\right)\right|} \geq 1
$$

and $t_{0}=t_{0}(z, t) \in B\left(z^{0}, t\right)$ is chosen in (16) and $\left|t_{0}-t\right| \leq 1 / L\left(z^{0}+t \mathbf{b}\right)$. From $\left|t^{\prime}-t_{0}\right|=\frac{2}{L\left(z^{0}+t \mathbf{b}\right)}$ one has $\left|t^{\prime}\right| \leq\left|t_{0}\right|+\frac{2}{L\left(z^{0}+t \mathbf{b}\right)} \leq|t|+\frac{3}{L\left(z^{0}+t \mathbf{b}\right)}$. Therefore, $\beta \geq 3$.

If $L \in Q$, then $\sup \left\{\frac{L\left(z^{0}+t_{0} \mathbf{b}\right)}{L\left(z^{0}+t \mathbf{b}\right)}:\left|t-t_{0}\right| \leq \frac{1}{L\left(z^{0}+t \mathbf{b}\right)}\right\} \leq \lambda_{\mathbf{b}}(1)$. This means that $L\left(z^{0}+t \mathbf{b}\right) \geq$ $\frac{L\left(z^{0}+t_{0} \mathbf{b}\right)}{\lambda_{\mathbf{b}}(1)}$. Using this inequality, we choose in $(23)$

$$
c:=\sup _{z^{0} \in A} \frac{\max \left\{\left|F\left(z^{0}+t^{\prime} \mathbf{b}\right)\right|:\left|t^{\prime}-t_{0}\right|=\frac{2 \lambda_{\mathbf{b}}(1)}{L\left(z^{0}+t_{0} \mathbf{b}\right)}\right\}}{\left|F\left(z^{0}+t_{0} \mathbf{b}\right)\right|} \geq 1
$$

with $t_{0}$ chosen in (16). Taking into account (22) and (23), one has

$$
\frac{\left|\partial_{\mathbf{b}}^{p} F\left(z^{0}+t \mathbf{b}\right)\right|}{p ! L^{p}\left(z^{0}+t \mathbf{b}\right)} \leq c \max \left\{\left|F\left(z^{0}+t^{\prime} \mathbf{b}\right)\right|:\left|t^{\prime}-t_{0}\right|=\frac{2 r}{L\left(z^{0}+t \mathbf{b}\right)}\right\}
$$


for all $n \in \mathbb{N} \cup\{\mathbf{0}\}, r \geq 0, z^{0} \in A, t \in \mathbb{D}_{z^{0}}$.

We differentiate (18) $p$ times, $p \geq N+1$, and then apply (15), (24) and (16)

$$
\begin{gathered}
\frac{\left|\partial_{\mathbf{b}}^{p} H\left(z^{0}+t \mathbf{b}\right)\right|}{p ! L^{p}\left(z^{0}+t \mathbf{b}\right)} \leq \frac{\left|\partial_{\mathbf{b}}^{p} G\left(z^{0}+t \mathbf{b}\right)\right|}{p ! L^{p}\left(z^{0}+t \mathbf{b}\right)}+\frac{|\varepsilon|\left|\partial_{\mathbf{b}}^{p} F\left(z^{0}+t \mathbf{b}\right)\right|}{p ! L^{p}\left(z^{0}+t \mathbf{b}\right)} \leq \\
\leq \alpha \max \left\{\frac{\left|\partial_{\mathbf{b}}^{k} G\left(z^{0}+t \mathbf{b}\right)\right|}{k ! L^{k}\left(z^{0}+t \mathbf{b}\right)}: 0 \leq k \leq N\right\}+ \\
+c|\varepsilon| \max \left\{\left|F\left(z^{0}+t^{\prime} \mathbf{b}\right)\right|:\left|t^{\prime}-t_{0}\right|=\frac{2 r}{L\left(z^{0}+t \mathbf{b}\right)}\right\} \leq \\
\leq(\alpha+c|\varepsilon|) \max \left\{\frac{\left|\partial_{\mathbf{b}}^{k} G\left(z^{0}+t \mathbf{b}\right)\right|}{k ! L^{k}\left(z^{0}+t \mathbf{b}\right)}: 0 \leq k \leq N\right\} .
\end{gathered}
$$

If $s \leq N$, then (24) is valid for $p=s$, but (15) does not hold. Thus, the differentiation of (18) leads to the following estimate

$$
\begin{gathered}
\frac{\left|\partial_{\mathbf{b}}^{s} H\left(z^{0}+t \mathbf{b}\right)\right|}{s ! L^{s}\left(z^{0}+t \mathbf{b}\right)} \geq \frac{\left|\partial_{\mathbf{b}}^{S} G\left(z^{0}+t \mathbf{b}\right)\right|}{s ! L^{s}\left(z^{0}+t \mathbf{b}\right)}-\frac{|\varepsilon|\left|\partial_{\mathbf{b}}^{s} F\left(z^{0}+t \mathbf{b}\right)\right|}{s ! L^{s}\left(z^{0}+t \mathbf{b}\right)} \geq \\
\geq \frac{\left|\partial_{\mathbf{b}}^{s} G\left(z^{0}+t \mathbf{b}\right)\right|}{s ! L^{s}\left(z^{0}+t \mathbf{b}\right)}-c|\varepsilon| \max \left\{\left|F\left(z^{0}+t^{\prime} \mathbf{b}\right)\right|:\left|t^{\prime}-t_{0}\right|=\frac{2 r}{L\left(z^{0}+t \mathbf{b}\right)}\right\},
\end{gathered}
$$

where $0 \leq s \leq N$. From (16) and (26) we deduce

$$
\max _{0 \leq s \leq N}\left\{\frac{\left|\partial_{\mathbf{b}}^{s} H\left(z^{0}+t \mathbf{b}\right)\right|}{s ! L^{s}\left(z^{0}+t \mathbf{b}\right)}\right\} \geq(1-c|\varepsilon|) \max _{0 \leq s \leq N}\left\{\frac{\left|\partial_{\mathbf{b}}^{s} G\left(z^{0}+t \mathbf{b}\right)\right|}{s ! L^{s}\left(z^{0}+t \mathbf{b}\right)}\right\} .
$$

If $c|\varepsilon|<1$, then (25) and (27) imply

$$
\frac{\left|\partial_{\mathbf{b}}^{p} H\left(z^{0}+t \mathbf{b}\right)\right|}{p ! L^{p}\left(z^{0}+t \mathbf{b}\right)} \leq \frac{\alpha+c|\varepsilon|}{1-c|\varepsilon|} \max _{0 \leq s \leq N}\left\{\frac{\left|\partial_{\mathbf{b}}^{s} H\left(z^{0}+t \mathbf{b}\right)\right|}{s ! L^{s}\left(z^{0}+t \mathbf{b}\right)}\right\}
$$

for $p \geq N+1$. Assume that $\frac{\alpha+c|\varepsilon|}{1-c|\varepsilon|} \leq 1$. Hence, $|\varepsilon| \leq \frac{1-\alpha}{2 c}$.

Let $N_{\mathbf{b}}\left(z^{0}+t \mathbf{b}, L, F\right)$ be the $L$-index in the direction $\mathbf{b}$ of the function $F$ at the point $z^{0}+t \mathbf{b}$, i.e. $N_{\mathbf{b}}\left(z^{0}+t \mathbf{b}, L, F\right)$ is the smallest number $m_{0}$ for which inequality (3) holds with $z=z^{0}+t \mathbf{b}$.

For $|\varepsilon| \leq \frac{1-\alpha}{2 c}$ validity of (28) means that for all $z^{0} \in A$ and every $t \in D_{z^{0}}$ such that $F\left(z^{0}+t \mathbf{b}\right) \neq 0$ the $L$-index in the direction $\mathbf{b}$ at the point $z^{0}+t \mathbf{b}$ does not exceed $N$, i.e., $N_{\mathbf{b}}\left(z^{0}+t \mathbf{b}, F, L\right) \leq N$.

If for some $z^{0} \in A \quad F\left(z^{0}+t \mathbf{b}\right) \equiv 0$, then we have $H\left(z^{0}+t \mathbf{b}\right) \equiv G\left(z^{0}+t \mathbf{b}\right)$ and $N_{\mathbf{b}}\left(z^{0}+t \mathbf{b}, F, L\right)=N_{\mathbf{b}}\left(z^{0}+t \mathbf{b}, G, L\right) \leq N$. Thus, $H(z)$ has bounded $L$-index in the direction b with $N_{\mathbf{b}}(H, L) \leq N$. It completes the proof of Theorem 10 .

Remark 2. Every analytic function $F: \mathbb{B}^{n} \rightarrow \mathbb{C}$ with $N_{\mathbf{b}}(F, L)=0$ satisfies inequality (17) (see proof of the necessity in [6, Theorem 2]).

If $L \in Q_{\mathbf{b}}\left(\mathbb{B}^{n}\right)$, then condition 2) in Theorem 10 always holds. The following theorem is valid.

Theorem 11. Let $L \in Q_{\mathbf{b}}\left(\mathbb{B}^{n}\right), \alpha \in(1 / \beta, 1)$ and $F, G$ be analytic functions in $\mathbb{B}^{n}$ which satisfy condition: 
1) $G(z)$ has bounded L-index in the direction $\mathbf{b} \in \mathbb{C}^{n} \backslash\{\mathbf{0}\}$.

2) for every $z=z^{0}+t \mathbf{b} \in \mathbb{B}^{n}$, where $z^{0} \in A$, and some $t_{0} \in B\left(z^{0}, t\right)$, and $r=\left|t-t_{0}\right| L\left(z^{0}+\right.$ $t$ b)

$$
\begin{aligned}
& \max \left\{\left|F\left(z^{0}+t^{\prime} \mathbf{b}\right)\right|:\left|t^{\prime}-t_{0}\right|=\frac{2 r}{L\left(z^{0}+t \mathbf{b}\right)}\right\} \leq \\
& \leq \max \left\{\frac{\left|\partial_{\mathbf{b}}^{k} G\left(z^{0}+t \mathbf{b}\right)\right|}{k ! L^{k}\left(z^{0}+t \mathbf{b}\right)}: 0 \leq k \leq N_{\mathbf{b}}\left(G_{\alpha}, L_{\alpha}\right)\right\}
\end{aligned}
$$

3) $c:=\sup _{z^{0} \in A} \frac{\max \left\{\left|F\left(z^{0}+t^{\prime} \mathbf{b}\right)\right|:\left|t^{\prime}-t_{0}\right|=\frac{2 \lambda_{2}^{\mathbf{b}}(1)}{L\left(z^{0}+t_{0} \mathbf{b}\right)}\right\}}{\left|F\left(z^{0}+t_{0} \mathbf{b}\right)\right|}<\infty$ where $t_{0}$ is chosen in 2).

If $|\varepsilon| \leq \frac{1-\alpha}{2 c}$, then the function $H(z)=G(z)+\varepsilon F(z)$ has bounded $L$-index in the direction b with $N_{\mathbf{b}}(H, L) \leq N_{\mathbf{b}}\left(G_{\alpha}, L_{\alpha}\right)$, where $G_{\alpha}(z)=G(z / \alpha), L_{\alpha}(z)=L(z / \alpha)$.

Proof. Condition 2) in Theorem 10 always holds for $N=N_{b}\left(G_{\alpha}, L_{\alpha}\right)$ instead of $N=$ $N_{\mathbf{b}}(G, L)$. Indeed by Theorem 9 inequality (14) is satisfied for the function $G$. Substituting $\frac{z^{0}}{\alpha}, \frac{t}{\alpha}$ and $\frac{t_{0}}{\alpha}$ instead $z^{0}, t$ and $t_{0}$ in (14) we obtain

$$
\begin{aligned}
& \max \left\{\left|G\left(\left(z^{0}+t \mathbf{b}\right) / \alpha\right)\right|:\left|t-t_{0}\right|=\frac{r_{2} \alpha}{L\left(\left(z^{0}+t_{0} \mathbf{b}\right) / \alpha\right)}\right\} \leq \\
\leq & P_{1} \max \left\{\left|G\left(\left(z^{0}+t \mathbf{b}\right) / \alpha\right)\right|:\left|t-t_{0}\right|=\frac{r_{1} \alpha}{L\left(\left(z_{0}+t_{0} \mathbf{b}\right) / \alpha\right)}\right\} .
\end{aligned}
$$

By Theorem 9 inequality (29) means that $G_{\alpha}=G(z / \alpha)$ has bounded $L_{\alpha}$-index in the direction $\mathbf{b}$ and vice versa. Then for $p \geq N_{\mathbf{b}}\left(G_{\alpha}, L_{\alpha}\right)+1$ and $\alpha \in(1 / \beta, 1)$

$$
\begin{aligned}
\frac{\left|\partial_{\mathbf{b}}^{p} G_{\alpha}(z)\right|}{p ! L_{\alpha}^{p}(z)}= & \frac{\left|\partial_{\mathbf{b}}^{p} G(z / \alpha)\right|}{p ! \alpha^{p} L^{p}(z / \alpha)} \leq \max \left\{\frac{\left|\partial_{\mathbf{b}}^{s} G_{\alpha}(z)\right|}{s ! L_{\alpha}^{s}(z)}: 0 \leq s \leq N_{\mathbf{b}}\left(G_{\alpha}, L_{\alpha}\right)\right\}= \\
& =\max \left\{\frac{\left|\partial_{\mathbf{b}}^{s} G(z / \alpha)\right|}{s ! \alpha^{s} L^{s}(z / \alpha)}: 0 \leq s \leq N_{\mathbf{b}}\left(G_{\alpha}, L_{\alpha}\right)\right\} .
\end{aligned}
$$

Multiplying by $\alpha^{p}$, we deduce

$$
\begin{gathered}
\frac{\left|\partial_{\mathbf{b}}^{p} G(z / \alpha)\right|}{p ! L^{p}(z / \alpha)} \leq \max \left\{\frac{\alpha^{p-s}\left|\partial_{\mathbf{b}}^{s} G(z / \alpha)\right|}{s ! L^{s}(z / \alpha)}: 0 \leq s \leq N_{\mathbf{b}}\left(G_{\alpha}, L_{\alpha}\right)\right\} \leq \\
\leq \alpha \max \left\{\frac{\left|\partial_{\mathbf{b}}^{s} G(z / \alpha)\right|}{s ! L^{s}(z / \alpha)}: 0 \leq s \leq N_{\mathbf{b}}\left(G_{\alpha}, L_{\alpha}\right)\right\} .
\end{gathered}
$$

Since $z$ is arbitrary, inequality (30) yields (15).

It is easy to see that $N_{\mathbf{b}}\left(G_{\alpha}, L_{\alpha}\right) \leq N_{\mathbf{b}}(G, L)$ for $\alpha \in(0,1)$. Thus, $N_{\mathbf{b}}\left(G_{\alpha}, L_{\alpha}\right)$ in Theorem 11 can be replaced by $N_{\mathbf{b}}(G, L)$.

Corollary 1. Let $l \in Q(\mathbb{D}), \alpha \in(1 / \beta, 1), \beta>\lambda(1)$ and $f, g$ be analytic functions in the unit disc $\mathbb{D}$, satisfying the conditions:

1) $g(z)$ has bounded l-index; 
2) for every $t \in \mathbb{C}$ there exists $t_{0}$ such that $f\left(t_{0}\right) \neq 0,\left|t_{0}-t\right|<\min \left\{\frac{\beta}{2 l(t)} ; \frac{1-|t|}{2}\right\}$ and for $r=\left|t-t_{0}\right| l(t)$ one has

$$
\max \left\{\left|f\left(t^{\prime}\right)\right|:\left|t^{\prime}-t_{0}\right|=\frac{2 r}{l(t)}\right\} \leq \max \left\{\frac{\left|g^{(k)}(t)\right|}{k ! l^{k}(t)}: 0 \leq k \leq N\left(g_{\alpha}, l_{\alpha}\right)\right\} .
$$

3) for all $t_{0}$ chosen in condition 2) one has $\max \left\{\left|f\left(t^{\prime}\right)\right|:\left|t^{\prime}-t_{0}\right|=\frac{2 \lambda(1)}{l\left(t_{0}\right)}\right\} /\left|f\left(t_{0}\right)\right| \leq c<$ $+\infty$.

If $|\varepsilon| \leq \frac{1-\alpha}{2 c}$, then the function $h(z)=g(z)+\varepsilon f(z)$ is of bounded l-index with $N(h, l) \leq$ $N\left(g_{\alpha}, l_{\alpha}\right)$, where $g_{\alpha}(z)=g(z / \alpha), l_{\alpha}(z)=l(z / \alpha)$.

Theorems 10 and 11 are new even for $n=1$, i.e. for analytic functions in the unit disc.

5. $L$-index in direction in a domain compactly embedded in the unit ball. Let $D$ be an arbitrary bounded domain in $\mathbb{B}^{n}$ such that $\operatorname{dist}\left(D, \partial \mathbb{B}^{n}\right)>0$. If inequality (3) holds for all $z \in D$ instead of $\partial \mathbb{B}^{n}$, then the analytic function $F: \partial \mathbb{B}^{n} \rightarrow \mathbb{C}$ is called a function of bounded $L$-index in the direction $\mathbf{b}$ in the domain $D$. The least such integer $m_{0}$ is called the $L$-index in the direction $\mathbf{b} \in \mathbb{C}^{n} \backslash\{\mathbf{0}\}$ in domain $D$ and is denoted by $N_{\mathbf{b}}(F, L, D)=m_{0}$. The notation $\bar{D}$ stands for a closure of the domain $D$.

Lemma 2. Let $D$ be an arbitrary bounded domain in $\mathbb{B}^{n}$ such that $d=\operatorname{dist}\left(D, \partial \mathbb{B}^{n}\right)=$ $\inf _{z \in D}(1-|z|)>0, \beta>1, \mathbf{b} \in \mathbb{C}^{n} \backslash\{\mathbf{0}\}$ be an arbitrary direction. If $L: \mathbb{B}^{n} \rightarrow \mathbb{R}_{+}$is continuous function such that $L(z) \geq \frac{\beta|b|}{d}$, and $F: \mathbb{B}^{n} \rightarrow \mathbb{C}$ is analytic function such that $\left(\forall z^{0} \in \bar{D}\right): \quad F\left(z^{0}+t \mathbf{b}\right) \not \equiv 0$, then $N_{\mathbf{b}}(F, L, D)<\infty$.

Proof. For every fixed $z^{0} \in \bar{D}$ we expand the analytic function $F\left(z^{0}+t \mathbf{b}\right)$ in a power series by powers of $t$ in the $\operatorname{disc}\left\{t \in \mathbb{C}:|t| \leq \frac{1}{L\left(z^{0}\right)}\right\}$

$$
F\left(z^{0}+t \mathbf{b}\right)=\sum_{m=0}^{\infty} \frac{\partial_{\mathbf{b}}^{m} F\left(z^{0}\right)}{m !} t^{m}
$$

The quantity $\frac{\left|\partial_{\mathbf{b}}^{m} F\left(z^{0}\right)\right|}{m !}$ is the modulus of a coefficient of the power series (31) at the point $t \in \mathbb{C}$ such that $|t|=\frac{1}{L\left(z^{0}\right)}$. Since $F(z)$ is function, for every $z_{0} \in \bar{D}$

$$
\frac{\left|\partial_{\mathbf{b}}^{m} F\left(z^{0}\right)\right|}{m ! L^{m}\left(z^{0}\right)} \rightarrow 0 \quad(m \rightarrow \infty),
$$

i.e., there exists $m_{0}=m\left(z^{0}, \mathbf{b}\right)$ such that inequality (3) holds at the point $z=z^{0}$ for all $m \in \mathbb{Z}_{+}$.

We prove that $\sup \left\{m_{0}: z^{0} \in \bar{D}\right\}<+\infty$. On the contrary we assume that the set of all values $m_{0}$ is unbounded in $z^{0}$, i.e., $\sup \left\{m_{0}: z^{0} \in \bar{D}\right\}=+\infty$. Hence, for every $m \in \mathbb{Z}_{+}$there exists $z^{(m)} \in \bar{D}$ and $p_{m}>m$

$$
\frac{1}{p_{m} ! L^{p_{m}}\left(z^{(m)}\right)}\left|\frac{\partial^{p_{m}} F\left(z^{(m)}\right)}{\partial \mathbf{b}^{p_{m}}}\right|>\max \left\{\frac{1}{k ! L^{k}\left(z^{(m)}\right)}\left|\frac{\partial^{k} F\left(z^{(m)}\right)}{\partial \mathbf{b}^{k}}\right|: 0 \leq k \leq m\right\} .
$$

Since $\left\{z^{(m)}\right\} \subset \bar{D}$, there exists a subsequence $z^{\prime(m)} \rightarrow z^{\prime} \in \bar{G}$ as $m \rightarrow+\infty$. By Cauchy's integral formula

$$
\frac{\partial_{\mathbf{b}}^{p} F(z)}{p !}=\frac{1}{2 \pi i} \int_{|t|=r} \frac{F(z+t \mathbf{b})}{t^{p+1}} d t
$$


for any $p \in \mathbb{N}, z \in D$. Rewrite (32) as following

$$
\begin{gathered}
\max \left\{\frac{1}{k ! L^{k}\left(z^{(m)}\right)}\left|\frac{\partial^{k} F\left(z^{(m)}\right)}{\partial \mathbf{b}^{k}}\right|: 0 \leq k \leq m\right\}< \\
<\frac{1}{L^{p_{m}}\left(z^{(m)}\right)} \int_{|t|=r / L\left(z^{(m)}\right)} \frac{\left|F\left(z^{(m)}+t \mathbf{b}\right)\right|}{|t|^{p_{m}+1}}|d t| \leq \frac{1}{r^{p_{m}}} \max \left\{|F(z)|: z \in D_{r}\right\},
\end{gathered}
$$

where $D_{r}=\bigcup_{z^{*} \in \bar{D}}\left\{z \in \mathbb{C}^{n}:\left|z-z^{*}\right| \leq \frac{|b| r}{L\left(z^{*}\right)}\right\}$. We can choose $r \in(1, \beta)$, because $F$ is a function analytic in the unit ball. Evaluating the limit for every directional derivative of fixed order in (33) as $m \rightarrow \infty$ we obtain

$$
\left(\forall k \in \mathbb{Z}_{+}\right): \quad \frac{1}{k ! L^{k}\left(z^{\prime}\right)}\left|\frac{\partial^{k} F\left(z^{\prime}\right)}{\partial \mathbf{b}^{k}}\right| \leq \varlimsup_{m \rightarrow \infty} \frac{1}{r^{p_{m}}} \max \left\{|F(z)|: z \in D_{r}\right\} \leq 0 .
$$

Thus, all derivatives in the direction $\mathbf{b}$ of the function $F$ at the point $z^{\prime}$ equals 0 and $F\left(z^{\prime}\right)=0$. In view of $(31) \quad F\left(z^{\prime}+t \mathbf{b}\right) \equiv 0$. It is a contradiction.

6. Existence theorem. We consider the function $F\left(z^{0}+t \mathbf{b}\right)$ where $z^{0} \in \mathbb{B}^{n}$ is fixed. If $F\left(z^{0}+t \mathbf{b}\right) \not \equiv 0$, then we denote by $p_{\mathbf{b}}\left(z^{0}+a_{k}^{0} \mathbf{b}\right)$ the multiplicity of the zero $a_{k}^{0}$ of the function $F\left(z^{0}+t \mathbf{b}\right)$. If $F\left(z^{0}+t \mathbf{b}\right) \equiv 0$ for some $z^{0} \in \mathbb{B}^{n}$, then we put $p_{\mathbf{b}}\left(z^{0}+t \mathbf{b}\right)=-1$.

Theorem 12. In order that for an analytic function $F: \mathbb{B}^{n} \rightarrow \mathbb{C}$ there exist a positive continuous function $L: \mathbb{B}^{n} \rightarrow \mathbb{R}_{+}$such that $F(z)$ is a function of bounded $L$-index in the direction $\mathbf{b}$ it is necessary and sufficient that $\exists p \in \mathbb{Z}_{+} \forall z^{0} \in \mathbb{B}^{n} \forall k p_{\mathbf{b}}\left(z^{0}+a_{k}^{0} \mathbf{b}\right) \leq p$.

Proof. Our proof is based on the proof for entire functions from [13] and for analytic functions in the unit ball of bounded L-index in joint variables from [10].

Necessity. To simplify the notation we consider everywhere in the proof $p_{k}^{0} \equiv p_{\mathbf{b}}\left(z^{0}+a_{0}^{k} \mathbf{b}\right)$. Necessity follows from the definition of analytic function of bounded $L$-index in direction. Indeed, assume on the contrary that $\forall p \in \mathbb{Z}_{+} \exists z^{0} \exists k p_{k}^{0}>p$. This means that

$$
\partial_{\mathbf{b}}^{p_{k}^{0}} F\left(z^{0}+a_{k}^{0} \mathbf{b}\right) \neq 0 \text { and } \partial_{\mathbf{b}}^{j} F\left(z^{0}+a_{k}^{0} \mathbf{b}\right)=0
$$

for all $j \in\left\{1, \ldots, p_{k}^{0}-1\right\}$. Therefore $L$-index in the direction $b$ at the point $z^{0}+a_{k}^{0} \mathbf{b}$ is not less than $p_{k}^{0}>p$

$$
N_{\mathbf{b}}\left(F, L, z^{0}+a_{k}^{0} \mathbf{b}\right)>p .
$$

If $p \rightarrow+\infty$, then we obtain that $N_{\mathbf{b}}\left(F, L, z^{0}+a_{k}^{0} \mathbf{b}\right) \rightarrow+\infty$. But this contradicts the boundedness of $L$-index in the direction of the function $F$.

Sufficiency. If for some $z^{0} \in \mathbb{B}^{n}, F\left(z^{0}+t \mathbf{b}\right) \equiv 0$, then inequality (3) is obvious.

Let $p$ be the smallest integer such that $\forall z^{0} \in \mathbb{B}^{n} F\left(z^{0}+t \mathbf{b}\right) \not \equiv 0$, and $\forall k p_{k}\left(z^{0}\right) \leq p$. For any point $z \in \mathbb{B}^{n}$ we define unambiguously the choice of $z^{0} \in \mathbb{C}^{n}$ and $t_{0} \in \mathbb{C}$ such that $z=z^{0}+t_{0} \mathbf{b}$. We choose a point $z^{0} \in \mathbb{B}^{n}$ on the hyperplane $\langle z, \mathbf{b}\rangle=0$, i.e. the point $z^{0}$ is a projection of point $z$ on the hyperplane. Therefore, there exists $t_{0} \in D_{z^{0}}$ such that $z=z^{0}+t \mathbf{b}$. Let $R \in\left(0, \frac{1-\left|z^{0}\right|}{|\mathbf{b}|}\right)$. We define $r_{0}=\frac{1}{2} \min \{1-R, R\}$. We put $K_{R}=\{t \in$ $\left.\mathbb{C}: R-r_{0} \leq|t| \leq R+r_{0}\right\}$ for all $R \in\left(0, \frac{1-\left|z^{0}\right|}{|\mathbf{b}|}\right)$ and

$$
m_{1}\left(z^{0}, R\right)=\min _{a_{k}^{0} \in K_{R}} \max _{0 \leq s \leq p}\left\{\frac{\left|\partial_{\mathbf{b}}^{s} F\left(z^{0}+a_{k}^{0} \mathbf{b}\right)\right|}{s !}\right\},
$$


where $a_{k}^{0}$ are zeros of the function $F\left(z^{0}+t \mathbf{b}\right)$.

Since $F$ is analytic, there exists $\varepsilon=\varepsilon\left(z^{0}, R\right)>0$ such that

$$
\frac{\left|\partial_{\mathbf{b}}^{s_{0}} F\left(z^{0}+t \mathbf{b}\right)\right|}{s_{0} !} \geq \frac{m_{1}\left(z^{0}, R\right)}{2}
$$

for some $s_{0}=s\left(a_{k}^{0}\right) \in\{0, \ldots, p\}$ and for all $t \in K_{R} \cap\left\{t \in \mathbb{C}:\left|t-a_{k}^{0}\right|<\varepsilon\left(R, z^{0}\right)\right\}$ and for all $k$. We denote $G_{\varepsilon}^{0}=\bigcup_{a_{k}^{0} \in K_{R}}\left\{t \in \mathbb{C}:\left|t-a_{k}^{0}\right|<\varepsilon\right\}, m_{2}\left(z^{0}, R\right)=\min \left\{\left|F\left(z^{0}+t \mathbf{b}\right)\right|:|t| \leq\right.$ $\left.R+r_{0}, t \notin G_{\varepsilon}^{0}\right\}$,

$$
Q\left(R, z^{0}\right)=\min \left\{\frac{m_{1}\left(R, z^{0}\right)}{2}, m_{2}\left(R, z^{0}\right), 1\right\}
$$

We take $R=\left|t_{0}\right|$. Then at least one of the numbers $\left|F\left(z^{0}+t_{0} \mathbf{b}\right)\right|,\left|\partial_{\mathbf{b}} F\left(z^{0}+t_{0} \mathbf{b}\right)\right|, \ldots$, $\frac{1}{p !}\left|\partial_{\mathbf{b}}^{p} F\left(z^{0}+t_{0} \mathbf{b}\right)\right|$ is not less than $Q\left(R, z^{0}\right)$ (respectively, $\left.\frac{1}{s_{0} !} \mid \partial_{\mathbf{b}}^{s_{0}} F\left(z^{0}+t_{0}\right) \mathbf{b}\right) \mid$ for $t_{0} \in G_{\varepsilon}^{0}$ and $\left|F\left(z^{0}+t_{0} \mathbf{b}\right)\right|$ for $\left.t \notin G_{\varepsilon}\right)$. Hence

$$
\max \left\{\frac{1}{j !}\left|\partial_{\mathbf{b}}^{j} F\left(z^{0}+t_{0} \mathbf{b}\right)\right|: 0 \leq j \leq p\right\} \geq Q\left(R, z^{0}\right) .
$$

On the other hand, for $\left|t_{0}\right|=R$ and $j \geq p+1$ Cauchy's inequality is valid

$$
\frac{1}{j !}\left|\partial_{\mathbf{b}}^{j} F\left(z^{0}+t_{0} \mathbf{b}\right)\right|=\left|\frac{1}{2 \pi i} \int_{\left|\tau-t_{0}\right|=r_{0}} \frac{F\left(z^{0}+\tau \mathbf{b}\right)}{\left(\tau-t_{0}\right)^{j+1}} d \tau\right| \leq \frac{1}{r_{0}^{j}} \max \left\{\left|F\left(z^{0}+\tau \mathbf{b}\right)\right|:|\tau| \leq R+r_{0}\right\} .
$$

We choose a positive continuous function $L(z)$ such that

$$
L\left(z^{0}+t_{0} \mathbf{b}\right) \geq \max \left\{\frac{\max \left\{1, \max \left\{\left|F\left(z^{0}+t \mathbf{b}\right)\right|:|\tau| \leq R+r_{0}\right\}\right\}}{Q\left(R, z^{0}\right) r_{0}^{2}}, \frac{\beta}{1-\left|z^{0}+t_{0} \mathbf{b}\right|}\right\}>1 .
$$

From (34) and (35) with $\left|t_{0}\right|=R$ and $j \geq 2 \cdot p$ we obtain

$$
\begin{aligned}
& \frac{\frac{1}{j ! L^{j}\left(z^{0}+t_{0} \mathbf{b}\right)} \cdot\left|\partial_{\mathbf{b}}^{j} F\left(z^{0}+t_{0} \mathbf{b}\right)\right|}{\max \left\{\frac{1}{k ! L^{k}\left(z^{0}+t_{0} \mathbf{b}\right)}\left|\partial_{\mathbf{b}}^{k} F\left(z^{0}+t_{0} \mathbf{b}\right)\right|: 0 \leq k \leq p\right\}} \leq \frac{L^{-j}\left(z^{0}+t_{0} \mathbf{b}\right)}{r_{0}^{j} Q\left(R, z^{0}\right) L^{-p}\left(z^{0}+t_{0} \mathbf{b}\right)} \times \\
& \times\left(\frac{\max \left\{1, \max \left\{\left|F\left(z^{0}+t_{0} \mathbf{b}\right)\right|:|\tau| \leq R+r_{0}\right\}\right\}}{Q\left(R, z^{0}\right) r^{2}}\right)^{j / 2} \leq L^{p-j / 2}\left(z^{0}+t_{0} \mathbf{b}\right) \leq 1 .
\end{aligned}
$$

Since $z=z^{0}+t \mathbf{b}$, we have

$$
\frac{\left|\partial_{\mathbf{b}}^{j} F(z)\right|}{j ! L^{j}(z)} \leq \max \left\{\frac{\left|\partial_{\mathbf{b}}^{k} F(z)\right|}{k ! L^{k}(z)}: 0 \leq k \leq p\right\} .
$$

In view of arbitrariness of $z$, the function $F$ has bounded $L$-index in the direction $\mathbf{b}$.

Acknowledgement. These researches are inspired by Prof. O. B. Skaskiv. Author cordially thanks him for his questions and interesting ideas which help these studies. 


\section{REFERENCES}

1. A.I. Bandura, Sum of entire functions of bounded L-index in direction, Mat. Stud., 45 (2016), №2, 149-158. doi: 10.15330/ms.45.2.149-158

2. A.I. Bandura, O.B. Skaskiv, Analytic functions in the unit ball and sufficient sets of boundedness of L-index in direction, Bukovyn. Mat. Zh., 6 (2018), №1-2, 13-20.

3. A.I. Bandura, N.V. Petrechko, Sum of entire functions of bounded index in joint variables, Electr. J. Math. Anal. Appl., 6 (2018), №2, 60-67.

4. A. Bandura, O. Skaskiv, Functions analytic in a unit ball of bounded $\mathbf{L}$-index in joint variables, J. Math. Sci., 227(1) (2017), 1-12. doi: 10.1007/s10958-017-3570-6

5. A.I. Bandura, O.B. Skaskiv, Analytic in an unit ball functions of bounded L-index in direction, 2015. arXiv: 1501.04166v2

6. A. Bandura, O. Skaskiv, Functions analytic in the unit ball having bounded L-index in a direction, to appear in Rocky Mountain J. Math. https://projecteuclid.org/euclid.rmjm/1542942029

7. A.I. Bandura, Analytic functions in the unit ball of bounded value L-distribution in a direction, Mat. Stud., 49 (2018), №1, 75-79. doi:10.15330/ms.49.1.75-79

8. A.I. Bandura, Product of two entire functions of bounded L-index in direction is a function with the same class, Bukovyn. Mat. Zh., 4 (2016), №1-2, 8-12.

9. A. Bandura, O. Skaskiv, Sufficient conditions of boundedness of L-index and analog of Hayman's Theorem for analytic functions in a ball, Stud. Univ. Babeş-Bolyai Math., 63 (2018), № 4, $483-501$. doi:10.24193/subbmath.2018.4.06

10. A. Bandura, O. Skaskiv, Analytic functions in the unit ball of bounded $\mathbf{L}$-index in joint variables and of bounded L-index in direction: a connection between these classes, Demonstratio Mathematica, 52 (2019), №1, 82-87. doi:10.1515/dema-2019-0008

11. A.I. Bandura, O.B. Skaskiv, Analytic functions in the unit ball of bounded L-index: asymptotic and local properties, Mat. Stud., 48 (2017), №1, 37-73. doi: 10.15330/ms.48.1.37-73

12. A.I. Bandura, O.B. Skaskiv, Entire functions of bounded L-index in direction, Mat. Stud., 27 (2007), №1, 30-52. (in Ukrainian)

13. A.I. Bandura, O.B. Skaskiv, Boundedness of L-index in direction of functions of the form $f(\langle z, m\rangle)$ and existence theorems, Mat. Stud., 41 (2014), №1, 45-52.

14. A.I. Bandura, O.B. Skaskiv, Boundedness of the L-index in a direction of entire solutions of second order partial differential equation, Acta Comment. Univ. Tartu. Math., 22 (2018), №2, 223-234. doi: 10.12697/ACUTM.2018.22.18

15. A. Bandura, O. Skaskiv, Entire functions of several variables of bounded index, Lviv: Publisher I. E. Chyzhykov, 2016, 128 p.

16. A. Bandura, Composition of entire functions and bounded L-index in direction, Mat. Stud., 47 (2017), №2, 179-184. doi:10.15330/ms.47.2.179-184

17. W.K. Hayman, Differential inequalities and local valency, Pacific J. Math., 44 (1973), №1, 117-137.

18. V.O. Kushnir, On analytic in a disc functions of bounded l-index, Visn. Lviv Un-ty, Ser. Mekh.-Math., 58 (2000), 21-24. (in Ukrainian)

19. V.O. Kushnir, Analytic function of bounded l-index: diss. ... Cand. Phys. and Math. Sciences, Ivan Franko National University of Lviv, Lviv, 2002, 132 p. (in Ukrainian)

20. A.D. Kuzyk, M.N. Sheremeta, Entire functions of bounded l-distribution of values, Math. Notes, 39 (1986), №1, 3-8. doi:10.1007/BF01647624

21. B. Lepson, Differential equations of infinite order, hyperdirichlet series and entire functions of bounded index, Proc. Sympos. Pure Math., 2 (1968), 298-307.

22. M.N. Sheremeta, Entire functions and Dirichlet series of bounded l-index, Russian Math. (Iz. VUZ), 36 (1992), №9, 76-82.

23. M.N. Sheremeta, A.D. Kuzyk, Logarithmic derivative and zeros of an entire function of bounded $l$-index, Sib. Math. J., 33 (1992), №2, 304-312. doi:10.1007/BF00971102

24. M. Sheremeta, Analytic functions of bounded index, Lviv: VNTL Publishers, 1999, $141 \mathrm{p}$.

25. S.N. Strochyk, M.M. Sheremeta, Analytic in the unit disc functions of bounded index. Dopov. Akad. Nauk Ukr., 1993, №1, 19-22. (in Ukrainian) 
26. W.J. Pugh, Sums of functions of bounded index, Proc. Amer. Math. Soc., 22 (1969), 319-323.

27. F. Nuray, R.F. Patterson, Vector-valued bivariate entire functions of bounded index satisfying a system of differential equations, Mat. Stud., 49 (2018), №1, 67-74, doi: 10.15330/ms.49.1.67-74

Department of Advanced Mathematics,

Ivano-Frankivsk National Technical University of Oil and Gas,

Ivano-Frankivsk, Ukraine

andriykopanytsia@gmail.com 